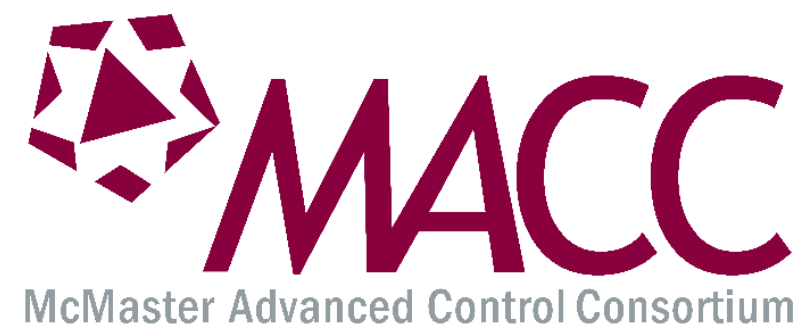

\title{
Article Pre-Print
}

The following article is a "pre-print" of an article accepted for publication in an Elsevier journal.

Meidanshahi, V., Corbett, B., Adams, T. A. II, Mhaskar, P. Subspace Model Identification and Model Predictive Control Based Cost Analysis of a Semicontinuous Distillation Process, in press (2017)

The pre-print is not the final version of the article. It is the unformatted version which was submitted for peer review, but does not contain any changes made as the result of reviewer feedback or any editorial changes. Therefore, there may be differences in substance between this version and the final version of record.

The final, official version of the article can be downloaded from the journal's website via this DOI link when it becomes available (subscription or purchase may be required):

\section{doi: 10.1016/j.compchemeng.2017.03.011}

This pre-print has been archived on the author's personal website (macc.mcmaster.ca) and/or the author's institutional repository (macsphere.mcmaster.ca) in compliance with the National Sciences and Engineering Research Council (NSERC) policy on open access and in compliance with Elsevier's academic sharing policies.

(C2017. This manuscript version is made available under the CC-BY-NC-ND 4.0 license http://creativecommons.org/licenses/by-nc-nd/4.0/

Date Archived: March 20, 2017 


\title{
Subspace Model Identification and Model Predictive Control of a Semicontinuous Distillation Process
}

\author{
Vida Meidanshahi, Brandon Corbett, Thomas A. Adams II*, Prashant Mhaskar \\ Department of Chemical Engineering, McMaster University, 1280 Main St. West, Hamilton, Ontario, \\ Canada, L8S $4 L 8$ \\ *Corresponding author. (tadams@mcmaster.ca), McMaster University, Department of Chemical \\ Engineering, 1280 Main St West, Hamilton, Ontario, Canada, L8S 4L8
}

\begin{abstract}
Semicontinuous distillation is a process intensification technique for purification of multicomponent mixtures. The system is control-driven and thus the control structure and its tuning parameters have crucial importance in the operation and the economics of the process. In this study, for the first time, implementation of the model predictive control (MPC) on a semicontinuous process is studied. A cascade configuration of MPC and PI controllers is designed in which the setpoints of the PI controllers are determined via a shrinking-horizon MPC. The objective is to reduce the operating cost of a cycle while simultaneously maintaining the required product qualities. A subspace identification method is adopted to identify a linear, state-space model to be used in the MPC. The first-principals model of the process is then simulated in gPROMS. Simulation results demonstrate that the MPC has reduced the operational cost of a semicontinuous process by about $11 \%$.
\end{abstract}

\section{Keywords:}

Semicontinuous distillation; Model predictive control (MPC); Cascade MPC with PI; Subspace identification; Dynamic distillation; gPROMS 


\section{Introduction}

Distillation columns are usually major sources of capital and operating costs in chemical processes (Dunnebier and Pantelides, 1999). Unsurprisingly, reducing these costs has been the driving force for intensive research on process intensification techniques aimed at the mitigation of these major costs. Among such studies are those that consider heat integration configurations aimed at reducing the operating costs of the process (Jana, 2010) and those that consider dividing wall columns that can potentially reduce the capital and operating costs of multicomponent separation (Yildirim et al., 2011). Semicontinuous distillation is another intensification technique that has been shown effective in reducing the total annualized cost (TAC) of processes with low to intermediate throughputs (Adams and Pascall, 2012). The semicontinuous process was first proposed by Phimister and Seider (2000) for ternary mixtures and was extended for $n$-component mixtures by Wijesekera and Adams (2015a and 2015b).

The essential idea in semicontinuous distillation is to lower the capital costs by purifying $n$ component mixtures in only one distillation column that is coupled with storage tanks (called middle vessels). Conventionally, $n-1$ distillation columns are required to separate an $n$ component mixture in a steady-state approach (Figure 1a). Alternatively, the same purification can be achieved dynamically with semicontinuous system. In this configuration, $n-2$ middle vessels are integrated with a single distillation column (Figure 1b).

A typical semicontinuous cycle proceeds as follows: Initially, the middle vessels are charged with fresh feed. Each middle vessel feeds the column and a side stream of the column is recycled back to it. During the processing mode, the lightest and the heaviest components, in terms of boiling points, are removed from the distillate and bottom streams of the distillation column, respectively. Each intermediate component is concentrated in a middle vessel based on its relative volatility. The most volatile intermediate component is concentrated in a middle vessel that receives the side stream from a tray closer to the column's condenser, and the heaviest intermediate component is accumulated in a middle vessel that receives the recycle from a tray closer to the reboiler of the column. When the desired purities of all intermediate components are achieved in each of the middle vessels, the processing mode terminates and the vessels are discharged. The products are collected before the middle vessels are refilled with fresh feed, marking the end of one cycle.

By omitting $n-2$ distillation columns, the semicontinuous system lowers the capital cost of a given separation process and consequently the TAC of the process. Being a compact and less expensive configuration, semicontinuous distillation is thus a desirable candidate for lowthroughput processes such as biofuel or pharmaceutical processing (Sultana and Kumar, 2012).

Since the dynamic variability of semicontinuous processes is very high, its performance is heavily dependent on its control system. Proper control can shorten the cycle time, increase the production rate and reduce the operating cost of the process, while improper control can disrupt the cycle and even result in instability of the column, leading to safety concerns and economic 
losses. Despite the obvious importance of semicontinuous control system design, very few studies have examined the use of advanced control techniques to improve the process' performance. So far, only proportional integral (PI) control structures have been implemented on this configuration.
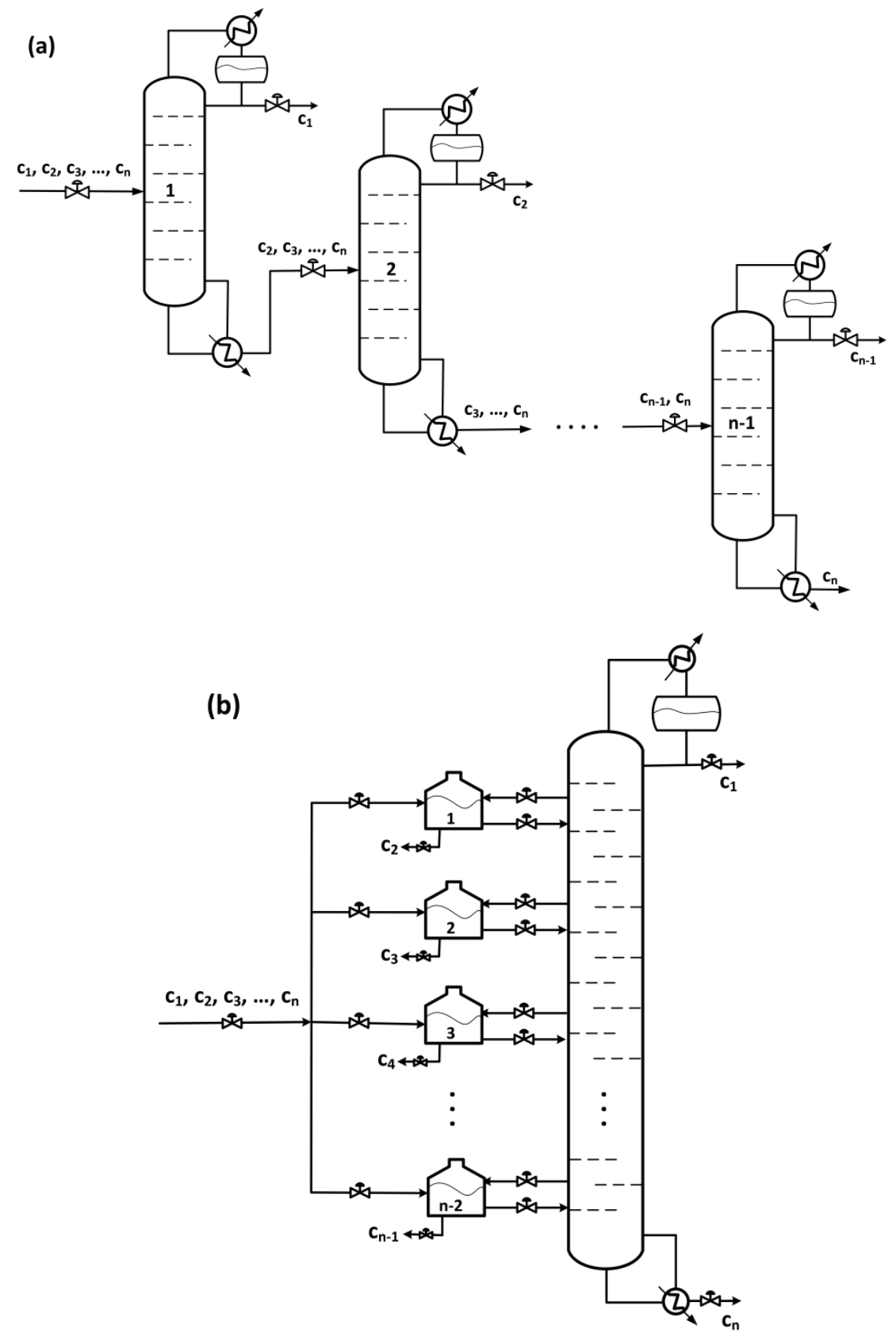

Figure 1- (a) conventional continuous distillation train for purification of $n$-component mixture, (b) the corresponding semicontinuous configuration. 
Pascall and Adams (2013) explored eight PI configurations for the semicontinuous system. They compared the configurations and evaluated their performances in terms of maintaining product purities and rejecting disturbances in the feed composition. They showed PI control can satisfactorily operate the system. However, the challenge with multi-PI controllers for semicontinuous distillation lies in tuning the controllers' parameters. High interactions between control loops are the main reason. Moreover, regular tuning methods such as Cohen-Coon, Ziegler-Nichols, Tyreus-Luyben and auto-tune rely on the assumption that the process is operating at steady-state. Since the semicontinuous system operates in a purely transient fashion and thus does not have any steady states, these methods cannot be used and a tedious trial-anderror procedure must be utilized.

To address this issue, Meidanshahi and Adams (2016) proposed an integrated design and control approach to simultaneously design the structural and operational parameters of the system. In their proposed method, the tuning parameters of the controllers are determined simultaneously with structural parameters (such as feed stream location, number of trays, etc.) in a mixed-integer dynamic optimization problem. Although this method provides a suitable methodology for tuning PI controllers, advanced control strategies such as the model predictive control (MPC) can likely provide more desirable control for a semicontinuous system. The MPC utilizes a model of the process, which allows it to optimize the inputs to the system to achieve a pre-defined objective (whether it is economic or operational) while considering for process constraints and dynamics. However, in large plants, MPC is not a replacement of proportional integral derivative (PID) controllers, but is rather an addition to it. While PID controllers have been shown to handle single-input/single-output systems effectively and have the benefit of not requiring process models, multi-input/multi-output processes and over-arching control objectives are better suited to being controlled via the MPC method.

There are several literature studies that feature the implementation of MPC on distillation columns. These studies have also investigated the specific way in which MPC is applied, such as whether it should be directly implemented on the column or used in combination with PI control loops.

For instance, Huang and Riggs (2002) studied the MPC control of a binary steady-state distillation column. They compared three control configurations for the system: In the first configuration, the MPC manipulated the reflux and bottoms flow rates to maintain the purities of distillate and bottom streams, respectively, and PI controllers were used for regulatory control of liquid levels in the reflux drum and sump by manipulating the distillate and boil up flow rates, respectively. In the second configuration, the MPC directly controlled all four degrees of freedom of the process which were the distillate, reflux, boilup and bottom flow rates. In the third configuration, a cascade design was studied in which the MPC was directly manipulating the reflux and bottom flow rates to control the product purities and indirectly controlling the liquid levels by manipulating the setpoints of the PI level controllers. The authors concluded that both the direct and cascade (the second and the third) configurations had desirable performances. 
In these scenarios, the MPC method could coordinate the input moves for all manipulated variables, whereas in the first configuration the MPC and the PI controllers had conflicting input actions to the system, leading to decreased performance. With respect to the performance of the MPC itself, the authors also discussed the advantages and disadvantages of direct versus cascade control configurations.

In the direct configuration, the MPC performed more reliably due to its independence of the PI regulatory level controllers. However, a significant disadvantage of this configuration was that the system could become unstable during step-tests for MPC model identification without the PI regulatory controls in place. The cascade configuration could compensate this shortcoming. High-fidelity models could be obtained with the cascade configuration while the MPC still had the leverage to manipulate the liquid levels by influencing the control input actions to the process via the PI setpoints.

The distillate and bottom composition loops in a distillation column usually have high levels of interaction with each other. Barolo and Papini (2000) suggested that the presence of a middle vessel can reduce this interaction and improve the performance of the column. They proposed a novel configuration called middle vessel continuous distillation column (MVCC) for binary mixtures. In this configuration, the feed stream was fed to the middle vessel, which had a level controller to adjust the feed flow rate to the column. The side stream of the column was recycled back to the middle vessel. The authors showed that the presence of a middle vessel could hydraulically decouple the interaction of the top and bottom composition loops and improve the composition control of the system. In this configuration, the choice of the middle vessel holdup volume and the level controller gain were the main design parameters of the system.

Subsequently, Bezzo et al. (2005) studied two MPC configurations of the MVCC. In the first, MPC was directly manipulating the five inputs to the system (distillate, reflux, bottom, boilup and feed flow rates). In the second configuration, the MPC manipulated the setpoints of three PI level controllers (controlling the reflux drum, sump and middle vessel liquid levels) while also directly controlling the reflux and boilup ratios. The authors used step tests to find a linear model of the process and implemented the MPC employing the MPC Toolbox of MATLAB. They concluded that by using MPC they could use a smaller middle vessel. However, it was also found that the MPC strategy had the same control performances as a properly tuned PI control structure and a proper middle vessel hold up.

On the other hand, Rewagad and Kiss (2012) observed outstanding performance of MPC on dividing wall columns compared to PID control. They also addressed the issue of direct or coupled MPC implementation. In the direct configuration, all inputs were computed by the MPC. In the other configuration, two PID controllers were used to control the liquid levels and the MPC was directly manipulating the other inputs to the system. A linearized model of the dividing wall column was used for calculations in the MPC. The authors observed that both MPC configurations showed similar performance and they could successfully lower the integral absolute error (IAE) of overshoots when compared to PID control. 
In this study, implementation of the MPC on a semicontinuous system is studied for the first time. Although the total direct cost of the semicontinuous system is lower than a conventional continuous system, its higher operating costs motivate the need for more effective cycle control. The higher operating costs are due to the fact that the side stream is continuously being recycled back to the middle vessel, increasing the entropy of the mixture by mixing the purified side stream with the non-purified hold up in the middle vessel. The objective of this work is to investigate if a semicontinuous process can be operated more efficiently and hence with lower operating costs, while still meeting the required product purities. In order to accomplish this objective, a subspace identification technique for batch processes (Corbett and Mhaskar, 2016) is adopted to identify a linear model of the semicontinuous distillation process and it is used in a shrinking-horizon MPC.

\section{Simulation Environment}

The separation of an equimolar ternary mixture of benzene, toluene and $o$-xylene (BTX) is chosen as a case study for this work. The semicontinuous purification of this mixture requires one middle vessel as shown in Figure 2. In this configuration, the distillate and bottom stream purities are controlled by their respective flow rates. The reflux drum and sump liquid levels are controlled by the feed flow rate to the column and the reboiler heat duty, respectively. Column pressure is maintained by manipulating the condenser heat duty. The flow rate of the side stream is adjusted by a flow rate controller. This control structure was shown to be the most effective multi-PI control loop for semicontinuous system in terms of disturbance rejection and maintaining product purities (Pascall and Adams, 2013). Therefore, this control structure is used in the present study for comparison against the proposed MPC alternative.

To simulate the process, gPROMS' process model library (PML) is used. PML provides firstprinciples models of commonly used process equipment and facilitates the simulation of process flowsheets. For details on simulating the BTX semicontinuous distillation in gPROMS, refer to Meidanshahi and Adams (2016). In the present work, the charging and discharging modes of the semicontinuous cycle are added to the simulation. The sequence of the operational modes is added to the SCHEDULE section of gPROMS. The differential algebraic equations (DAEs) of the system are integrated until the desired purity in the middle vessel is reached. Subsequently, the middle vessel discharge valve is opened to empty the tank until the liquid level reaches about $6 \%$ of the tank height. Following that, that valve is closed and the feed stream valve to the tank is opened. The middle vessel is then charged with fresh feed up to $80 \%$ of its height, thus beginning a new cycle. 


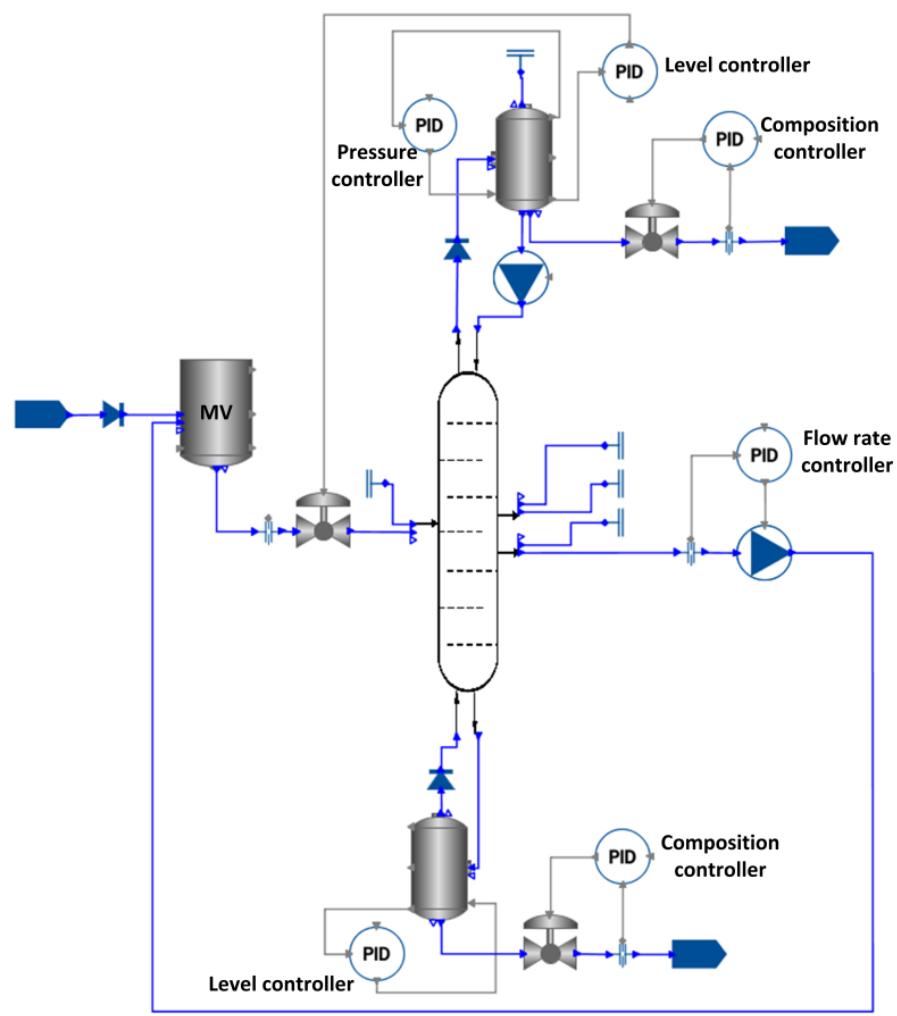

Figure 2- Semicontinuous distillation of BTX, simulated in gPROMS' PML.

\section{Implementation of MPC on Semicontinuous System}

MPC can be implemented on a semicontinuous system either directly or cascaded with PI control loops. The direct MPC implementation is shown in Figure 3. In this configuration, the column pressure and the sump liquid level are left to be controlled by PI controllers to ensure the stability of the process. However, the distillate, bottom, side stream and feed flow rates are directly manipulated by the MPC. The advantages of this configuration are elimination of the PI controllers and their dynamics from the control system. Moreover, this configuration eliminates the need for tedious trial-and-error tuning of the PI controllers. However, the disadvantage of this configuration is that the system is open-loop during the model identification step required for the MPC.

As the pseudo-random binary sequence (PRBS) signals are added to the nominal input trajectories (flow rates in this case) for the model identification, the outputs (composition of the products and constraint variables) deviate away from the closed-loop trajectories. Even with small PRBS amplitudes, the deviation was found to be significant which resulted in poor model identification. Not being able to identify a high-fidelity predictive model, the direct MPC configuration was ruled out for this study. 


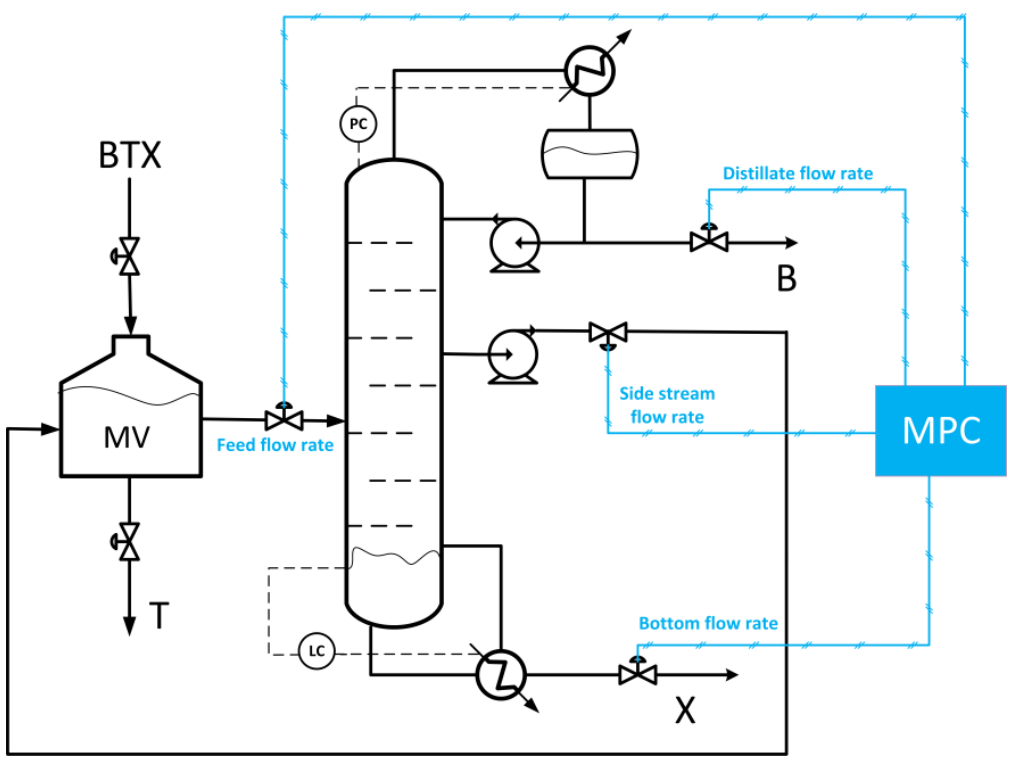

Figure 3- Direct MPC implementation on a semicontinuous system.

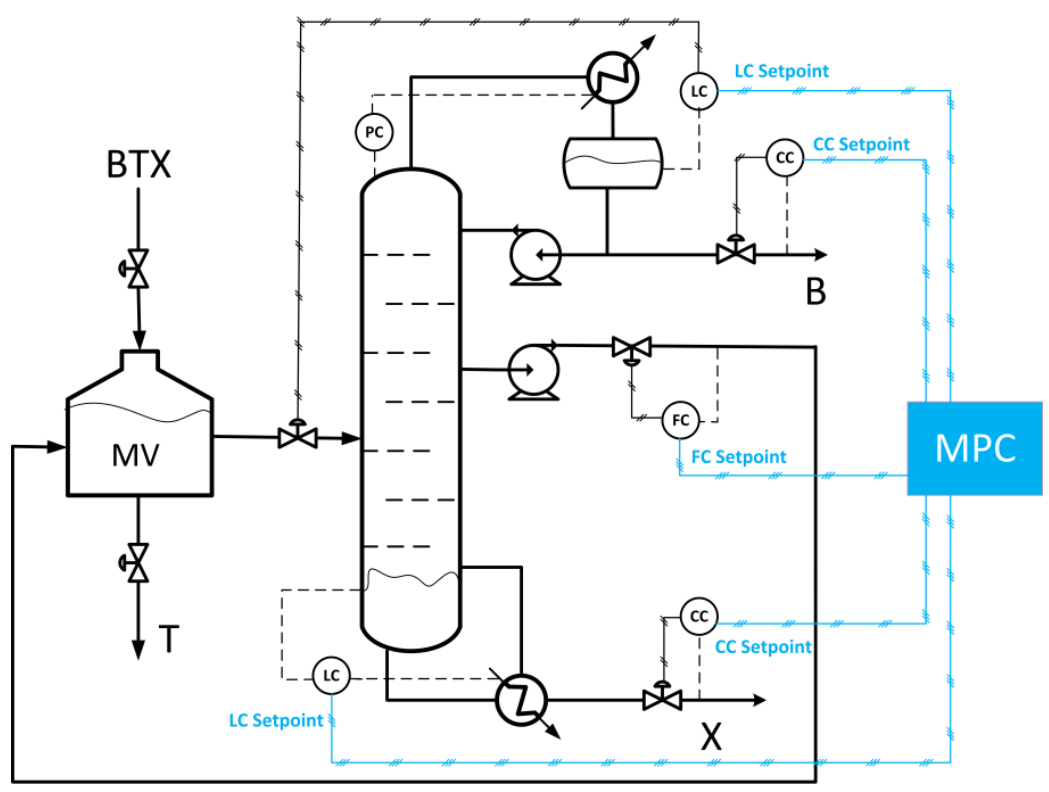

Figure 4- Cascaded MPC with multi-PI loops.

Therefore, the cascade MPC configuration is the next best option for the semicontinuous system (Figure 4). In this configuration, PI controllers ensure stability of the process. Also, during the model identification tests, the system remains in closed-loop. In this cascade configuration, the MPC adjusts the setpoints of the distillate and bottoms composition controllers, the side stream flow rate controller and the reflux drum and sump liquid level controllers. The column pressure controller setpoint is not considered as an input determined by the MPC since the column is 
designed to operate at the lowest possible pressure such that inexpensive cooling water could be used as the utility in the condenser. Further reducing the pressure would require the use of refrigerants, which are significantly more expensive.

The methodology presented by Corbett and Mhaskar (2016) for controlling batch processes is adopted here. They presented a subspace quality model predictive control (SQMPC) to obtain the desired product qualities by batch termination. The subspace identification method was used to identify a data-driven linear model of the process. Then they built a quality model which related the states and the inputs of the system to the quality variables. Quality variables were defined as process variables that are required to meet a desired specification by the end of the cycle or the batch. Having the process linear model and the quality model, the objective of the proposed shrinking horizon SQMPC was to reject disturbances in the batch initial condition and to track setpoint changes such that by the end of the batch product specifications were met. The main thrust of SQMPC is described next.

Consider a general dynamic process of the form:

$\dot{\boldsymbol{x}}=f(\boldsymbol{x}, \boldsymbol{u})$

$\boldsymbol{y}=g(\boldsymbol{x}, \boldsymbol{u})$

$\boldsymbol{q}=h(\boldsymbol{x}, \boldsymbol{u})$

where $\boldsymbol{x}$ is the state variable, $\boldsymbol{u}$ is the input, $\boldsymbol{y}$ is the output and $\boldsymbol{q}$ is a vector of quality variables. The nonlinear functions describing the dynamics of the system are denoted by $f, g$ and $h$ and are nonlinear functions relating the evolution of the process, outputs and quality variables, respectively, to the states and the inputs. Quality variables are defined as a subset of outputs that are required to meet a desired specification by the end of the cycle. They can be measured online or at the end of a batch. In the work of Corbett and Mhaskar (2016), the quality variables were unmeasured online and a data driven model was developed to predict them during the batch.

A full cycle of a semicontinuous process consists of charging, processing and discharging modes of operation. To simplify the control problem and avoid considering the charging and discharging phases in the MPC, a model is identified only for the processing mode of the system and the MPC is implemented only during that mode of operation. During the charging and discharging of the middle vessel, the MPC is turned off and the setpoints of all controllers are reset to their nominal values during the transitions. After recharging the middle vessel for the following cycle, the MPC is turned on again.

To design the MPC for semicontinuous system, it should be noted that the control system of the process should satisfy the following objectives:

1) The time-averaged purities of the distillate and bottom streams should meet the required specifications for these streams by the end of the processing mode. 
2) The desired purity of the intermediate component in the middle vessel must be achieved by the end of the processing mode.

3) The purification is performed in the shortest possible cycle time which results in increasing the total production rate and reducing the operating cost of the process.

4) The column pressure and liquid levels in the reflux drum and the sump are maintained at or close to their nominal values during the cycle to ensure stable operation.

5) Weeping and flooding should be avoided in the column during the cycle.

Therefore, the quality variables of the semicontinuous system are chosen to be the middle vessel purity $\left(q_{1}\right)$, the average purities of distillate $\left(q_{2}\right)$ and bottom $\left(q_{3}\right)$ streams, and the operating cost $\left(q_{4}\right)$. The product purities are set by design specifications and are required to be obtained by the end of the processing mode of the semicontinuous cycle. The time-averaged purities of distillate and bottom streams are computed via the following equations:

$q_{2}=\frac{\int_{0}^{t_{f}} F^{d i s} \times x_{B}^{d i s}}{\int_{0}^{t_{f}} F^{d i s}}$

$q_{3}=\frac{\int_{0}^{t_{f}} F^{b o t} \times x_{X}^{b o t}}{\int_{0}^{t_{f}} F^{b o t}}$

where $F$ is the flow rate of distillate and bottom streams and $x$ is the composition of the main component in those streams. The average purities are integrated from the beginning of the processing mode until the end of it $\left(t_{f}\right)$. The operating cost is also chosen as a quality variable since it is desired that the system operates with minimum energy consumption by the end of cycle. The operational cost is the cost of utilities in the condenser and the reboiler and is computed via Eq. 6. The costs of utilities ( $v_{1}$ and $v_{2}$ ) are computed based on the method available in Towler and Sinnott (2012).

$q_{4}=v_{1} \int_{0}^{t_{f}} Q_{\text {condenser }}+v_{2} \int_{0}^{t_{f}} Q_{\text {reboiler }}$

The output measurements $(y)$ of the semicontinuous system, which are considered in the MPC, are the reflux drum and sump liquid levels and the vapour velocities at top and bottom of the column (tray numbers 5 and 20, respectively). These measurements are needed to be considered in the MPC calculations to set operational constraints on the process to guarantee safe operation and avoid weeping and flooding of the column during the cycles. Furthermore, as with previous studies, it is assumed that the measurements of the quality and output variables are available free of measurement noise during the cycle. The inputs to the system $(\boldsymbol{u})$ are the setpoints of controllers as shown in Figure 4. The $\boldsymbol{f}, \boldsymbol{g}$ and $\boldsymbol{h}$ functions are the first-principles DAEs of the process which are simulated in gPROMS. 
The next step is to identify a discrete, time invariant, linear, state-space model. This model is employed in the suggested shrinking-horizon MPC, to predict the quality and the output variables during the cycle. In the following section, the procedure for identifying the model is discussed.

\section{Subspace Model Identification}

MPC computes future input moves to the process based on the provided model of the process. Therefore, the model should be both descriptive of the process dynamics and simple enough to allow for reasonable computation times of the optimization problem. Step-test identification methods cannot be used for a semicontinuous system due to the lack of any steady-state conditions of the process, which are required to identify process gains and time delays. Linearizing the nonlinear distillation column model is another option. In this method, multiple linear models are constructed along the process trajectory. These models could then be combined into one global model to describe the whole cycle, utilizing proper weighting factors (see, Aumi and Mhaskar, 2012 for an application to batch processes).

However, the subspace identification method is an alternative approach which can identify one linear model for the whole cycle and eliminate the need of weighting factors (Qin, 2006). This method is chosen to identify the linear model in this work. This data-driven method is based on gathering the input-output trajectories of the process and constructing a linear model (Moonen and De Moor, 1989). Corbett and Mhaskar (2016) expanded the method to apply to batch processes with varying batch length.

To adopt the Corbett and Mhaskar (2016) technique for the semicontinuous system, PRBS signals are added to the five inputs of the system (distillate and bottom composition controller setpoints, side stream flow rate setpoint, reflux drum and sump liquid level controller setpoints). The magnitudes of the PRBS signals are chosen to be significant enough to be able to identify the correlation between the inputs and the outputs. However, larger amplitudes result in poorly identified models.

Given the input and output trajectories of the process, the subspace identification method determines the $\boldsymbol{A}, \boldsymbol{B}, \boldsymbol{C}$ and $\boldsymbol{D}$ matrices of the state space model:

$\widetilde{\boldsymbol{x}}(k+1)=\boldsymbol{A} \widetilde{\boldsymbol{x}}(k)+\boldsymbol{B} \boldsymbol{u}(k)$

$y(k)=\boldsymbol{C} \widetilde{\boldsymbol{x}}(k)+\boldsymbol{D} \boldsymbol{u}(k)$

in which $\widetilde{\boldsymbol{x}}$ is the vector of subspace states which are different from the true states of the system. Therefore, a Luenberger observer should be deigned to obtain the true value of the states during the cycle. It was found that forcing the matrix $\boldsymbol{D}$ to zero for the present application resulted in a better model. Sampling time $(k)$ is considered to be one minute in this work which is in the order 
of process time scale. The identified model captures the dynamics of the PI controllers. However, it should be noted that the PI controllers should be tuned to have fast responses toward setpoint changes. This remark will be discussed more in section 6.2.

\section{SQMPC}

Once the linear model is obtained, the values of quality and output variables can be computed during the cycle and their terminal values can be predicted by the end of cycle for a candidate input profile. To do so, the values of the model states at each sampling time and the future input trajectories to the process should be known. To estimate the subspace model states, a Luenberger observer is utilized. As the process measurements become available, the observer is updated until the output predictions converge to the measured outputs. After the predicted outputs converge, the model predictive controller is employed in the closed-loop. The quality variables are computed at the end of the processing mode via the following equations:

$$
\begin{aligned}
& \tilde{\boldsymbol{x}}\left(k_{f}\right)=\boldsymbol{A}^{k_{f}-k} \tilde{x}(k)+\left[\begin{array}{llll}
\boldsymbol{A}^{k_{f}-k-1} \boldsymbol{B} & \boldsymbol{A}^{k_{f}-k-2} \boldsymbol{B} & \ldots & \boldsymbol{B}
\end{array}\right] \boldsymbol{v}(k) \\
& \boldsymbol{v}(k)=\left[\begin{array}{lll}
\boldsymbol{u}(k), & \boldsymbol{u}(k+1), \quad \ldots, \boldsymbol{u}\left(k_{f}-1\right)
\end{array}\right]^{\prime}
\end{aligned}
$$

where $k$ is the current sampling time and the $k_{f}$ is the final sampling instance. $v$ is the candidate input trajectory to the process. Subsequently, the quality variables can be readily computed as:

$$
\boldsymbol{q}\left(k_{f}\right)=\boldsymbol{C} \widetilde{\boldsymbol{x}}\left(k_{f}\right)
$$

Therefore, by having the predictions of the quality variables at the end of the cycle, the following MPC objective function can be computed and the future input trajectories to the process are optimized at each sampling time via a quadratic optimization problem:

$$
\min _{\boldsymbol{u}}\left(\boldsymbol{q}\left(k_{f}\right)-\boldsymbol{q}_{d}\right)^{\prime} \boldsymbol{M}\left(\boldsymbol{q}\left(k_{f}\right)-\boldsymbol{q}_{d}\right)+\Delta \boldsymbol{v}(k)^{\prime} \boldsymbol{P} \Delta \boldsymbol{v}(k)
$$

Subject to:

$$
\begin{aligned}
& \boldsymbol{q}=\boldsymbol{C}\left(\boldsymbol{A}^{k_{f}-k} \tilde{x}(k)+\left[\begin{array}{llll}
\boldsymbol{A}^{k_{f}-k-1} \boldsymbol{B} & \boldsymbol{A}^{k_{f}-k-2} \boldsymbol{B} & \ldots & \boldsymbol{B}
\end{array}\right] \boldsymbol{v}(k)\right) \\
& \Delta \boldsymbol{v}(k)=\left[\boldsymbol{u}(k), \boldsymbol{u}(k+1), \ldots, \boldsymbol{u}\left(k_{f}-1\right)\right]^{\prime}-\left[\boldsymbol{u}(k-1), \boldsymbol{u}(k), \ldots, \boldsymbol{u}\left(k_{f}-2\right)\right]^{\prime} \\
& \boldsymbol{v}_{\min }(k) \leq \boldsymbol{v}(k) \leq \boldsymbol{v}_{\max }(k)
\end{aligned}
$$


$V_{\min }^{i} \leq V^{i} \leq V_{\max }^{i}, \quad i=$ tray number 5 and 20

where $\boldsymbol{q}_{d}$ is the desired values of quality variables at the end of the processing mode. The diagonal matrix $\boldsymbol{M}$ penalizes any deviation from the desired qualities and the diagonal matrix $\boldsymbol{P}$ penalizes the magnitude of input moves. There are hard constraints on the inputs of the process and also on the outputs. The last constraint (Eq. 17) is intended to prevent weeping and flooding during the cycle. $V$ is the vapour velocity and the maximum allowable vapour velocity in the column to prevent flooding and the minimum vapour velocity to prevent weeping of liquid are computed based on equations provided in Mersmann et al. (2011).

The gPROMS rigorous first principle model is considered as the process model. The shrinking horizon MPC code is implemented in MATLAB. Therefore, the gO:MATLAB feature of gPROMS is used to link these two software platforms. The inputs to the process are computed in MATLAB and then they are passed into gPROMS, where the outputs of the process are computed and are sent back to MATLAB.

\section{Results and Discussion}

The structural parameters of the BTX semicontinuous separation used in this work are listed in Table 1, which are adopted from Meidanshahi and Adams (2016). In that work, the authors optimized the process and found the optimum structural and operational parameters for the system. However, they only considered the processing mode of operation in the optimization problem and did not include the charging and discharging modes. The reported gains for the side stream flow and liquid level controllers were small. When MPC is cascaded with those sluggish underlying PI controllers, the process does not respond quickly enough to the setpoint changes instructed by the MPC, and therefore the MPC changes the operation of the system by only a small amount. As a result, the improvement in the operational cost of the process is small ( 1$2 \%$ reduction in cost).

Table 1-Semicontinuous design parameters for BTX separation.

\begin{tabular}{cccccc}
\hline $\begin{array}{c}\text { Number of } \\
\text { trays }\end{array}$ & Feed tray & Side tray & $\begin{array}{c}\text { Condenser pressure } \\
\text { (bar) }\end{array}$ & $\begin{array}{c}\text { Middle vessel } \\
\text { height }(\mathrm{m})\end{array}$ & $\begin{array}{c}\text { Column diameter } \\
\text { (ft.) }\end{array}$ \\
\hline 26 & 16 & 13 & 0.37 & 5 & 4 \\
\hline
\end{tabular}


Another issue is that in this study the MPC is only on during the processing mode of the process and all controllers' setpoints are set back to their nominal values during the discharging and charging modes. Therefore, if the PI controllers are not fast enough to bring the process variables back to the their nominal values, the column will face material imbalance which results in liquid accumulation in the column or dry the column trays and eventually disrupts the operation of the process.

Therefore, the PI tuning parameters are changed to the values reported in Table 2 to avoid the aforementioned issues. Subsequently, the performances of the multi-PI loops and the cascaded MPC with PI controllers are compared. Furthermore, the charging and discharging rates of the middle vessel are designed at lower rates to give the process enough time for transition to the nominal conditions at the beginning of the subsequent cycle.

Table 2- PI controllers tuning parameters.

\begin{tabular}{|c|c|c|c|c|c|c|c|c|c|c|c|}
\hline \multicolumn{2}{|c|}{$\begin{array}{l}\text { Distillate Purity } \\
\text { controller }\end{array}$} & \multicolumn{2}{|c|}{$\begin{array}{c}\text { Bottom } \\
\text { Purity } \\
\text { controller }\end{array}$} & \multicolumn{2}{|c|}{$\begin{array}{l}\text { Reflux drum } \\
\text { level controller }\end{array}$} & \multicolumn{2}{|c|}{$\begin{array}{l}\text { Sump level } \\
\text { controller }\end{array}$} & \multicolumn{2}{|c|}{$\begin{array}{l}\text { Side draw } \\
\text { controller }\end{array}$} & \multicolumn{2}{|c|}{$\begin{array}{l}\text { Column } \\
\text { pressure } \\
\text { controller }\end{array}$} \\
\hline $\mathrm{k}$ & $\tau$ & $\mathrm{k}$ & $\tau$ & $\mathrm{k}$ & $\tau$ & $\mathrm{k}$ & $\tau$ & $\mathrm{k}$ & $\tau$ & $\mathrm{k}$ & $\tau$ \\
\hline 5 & 40200 & 5 & 50251 & 3 & 1000 & 3 & 1000 & 3 & 1000 & 0.5 & 1000 \\
\hline
\end{tabular}

$\mathrm{k}$ is the gain and $\tau$ is the reset time (sec.).

In the following subsections, first the results of model identification are discussed. Subsequently, performance of the MPC strategy on the semicontinuous system is examined in two case studies. The first case study explores the economic benefits of the MPC implementation on the process. The main focus in this case is to reduce the operating cost of a semicontinuous cycle. The second case study examines the capability of the MPC in maintaining product purities in the face of changes in the feed stock composition.

\subsection{Subspace Model}

As mentioned earlier, a linear model is identified only for the processing mode of the operation. A database of input-output trajectories of the process is generated to build the model. The database contains the nominal PI trajectories as well as the data gathered from exciting the process by adding PRBS signals to the setpoints of PI controllers. No open-loop data is collected in the database since it adversely affected the accuracy of the identified model.

Twenty percent of the database is used in model validation and the rest in training the model. The main parameters of the subspace identification method that should be determined are the number of states and the number of Hankle matrix rows. These parameters are chosen such that the sum square of errors of model predictions and process output measurements are minimized. These values for the identified model are 12 states and 14 Hankle rows. 
Figure 5 illustrates the prediction capabilities of the identified linear model. In this Figure the model predictions of the quality variables are compared to the corresponding process measurements. For the first eighty sampling instances, the Luenberger observer is in closed-loop and is updated with process measurements until the observer converges and the true values of states are obtained. Subsequently, the observer is turned off and model predictions are started with the obtained states from the observer. The inputs to the process are fed to the identified model and the quality variables are computed. Figure 5 shows that the identified model can predict the quality variables with sufficient precision.
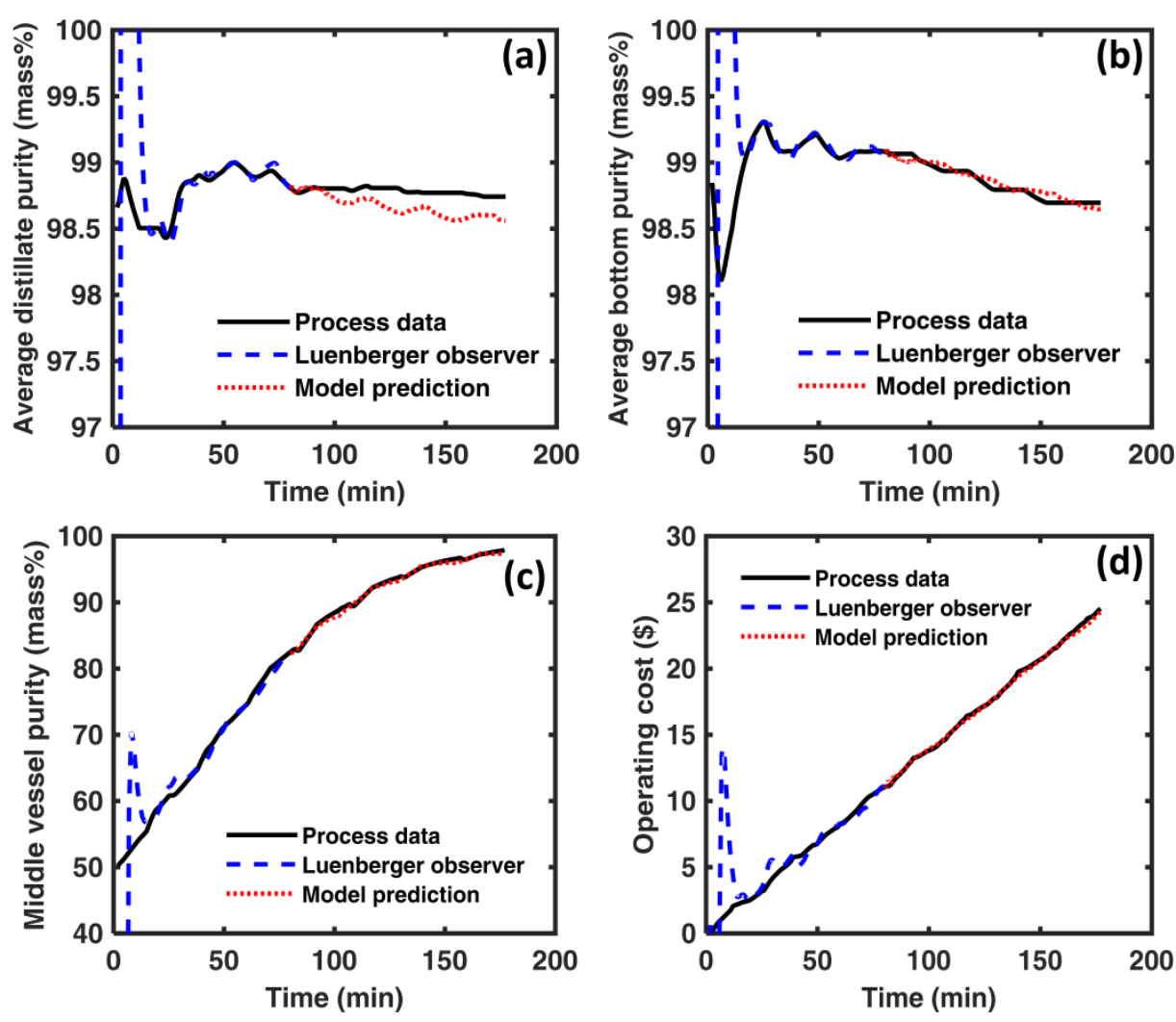

Figure 5- Comparison of the process quality variables measurements with the corresponding model predictions.

\subsection{Case 1: Cost improvement}

The emphasis of this case study is to explore the possibility of improving semicontinuous economics by implementing the MPC on the process. The goal here is to reduce the operating cost of the system while maintaining all product purities. To apply the MPC to the process, first the desired values of the quality variables that are required to be achieved by the end of the processing mode should be set. 
It should be noted that it takes a couple of cycles before the semicontinuous process converges to stabilized cycles. Therefore, in this work, the first ten cycles of the process with PI control are discarded and the nominal PI trajectories are considered from the eleventh cycle. The timeaverage purities of the distillate and bottom streams by the end of the processing mode of the eleventh cycle are 98.6 and 98.8 mass\%, respectively. These are selected as the desired final quality variable values. The other quality variable is the middle vessel purity and its desired final value is set to be 98 mass\%. Finally, the last quality variable is the operating cost of the process. The terminal value of this variable is not known beforehand and it is desired to be minimized. Therefore, any small value can be selected for it. In this work, it is set to be $80 \%$ of the operating cost of the process with PI control configuration.

The tuning parameters of the MPC, namely the $\boldsymbol{M}$ and $\boldsymbol{P}$ matrices, are tuned manually to obtain the best performance of the process. The chosen values for the diagonal elements of the $\boldsymbol{M}$ matrix are presented in Table 3. The values in this Table are the weights on the purity of the middle vessel $\left(m_{1}\right)$, the average purities of the distillate $\left(m_{2}\right)$ and bottom $\left(m_{3}\right)$ streams and the operating cost $\left(m_{4}\right)$. In regards with the $\boldsymbol{P}$ matrix, the penalty values of 100 are considered for the distillate and bottom setpoints moves and 10 for the rest of the input moves.

Table 3-Tuning parameters of the MPC for case 1.

\begin{tabular}{cccc}
\hline$m_{l}$ & $m_{2}$ & $m_{3}$ & $m_{4}$ \\
\hline 50 & 50 & 50 & 1000 \\
\hline
\end{tabular}

Figure 6 shows the PI controllers' setpoints during five full cycles of the semicontinuous system with PI-only and cascaded MPC control. During the discharge and recharge of the middle vessel the inputs are set back to the nominal values. Subsequently, the nominal inputs are implemented to the system until the observer converges (about 20 sampling times). After the convergence of the observer, the model predictions are started and the MPC is turned on. 

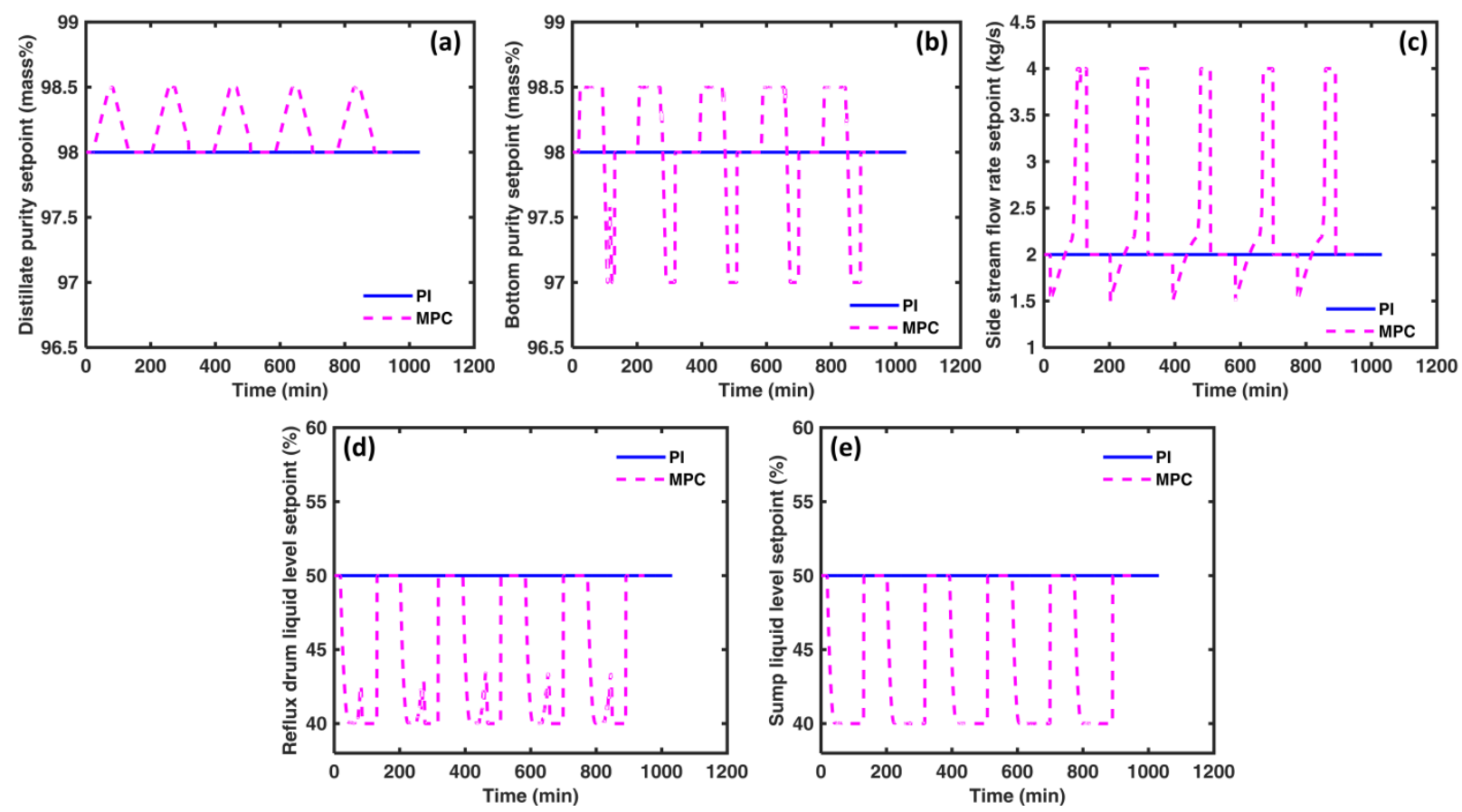

Figure 6- The PI controllers' setpoints in the PI-only and cascaded MPC control over the course of five cycles.

The quality variables are shown in Figure 7. For the closed-loop system under the PI controller alone, higher product purities (than the setpoints) are observed during the cycles (Figure 7a and 7b) which result in unnecessarily higher operating costs. This is a result of the underlying PI composition controllers being unable to regulate to the setpoint (see Figure 11b and 12b), which in turn results in taking more cycles before stable cycles are established. The cycles will eventually stabilize if no disturbances are considered to the system. Figure 7 shows the cycles from the $11^{\text {th }}$ until the $15^{\text {th }}$ cycles. The MPC is implemented starting in cycle 11 , which is time zero in this work.

Figure 7a, 7b and 7c show the average purities of the distillate and bottom streams and middle vessel purity, respectively. The MPC has obtained the desired product purities for these quality variables by the end of the processing modes. It has also stabilized the cycles and has cancelled the effect of the PI controllers that tend to increase the purities in each cycle. This is promising that the MPC can compensate the deficiencies of the imperfect tuning parameters of the PI controllers to some extent. 

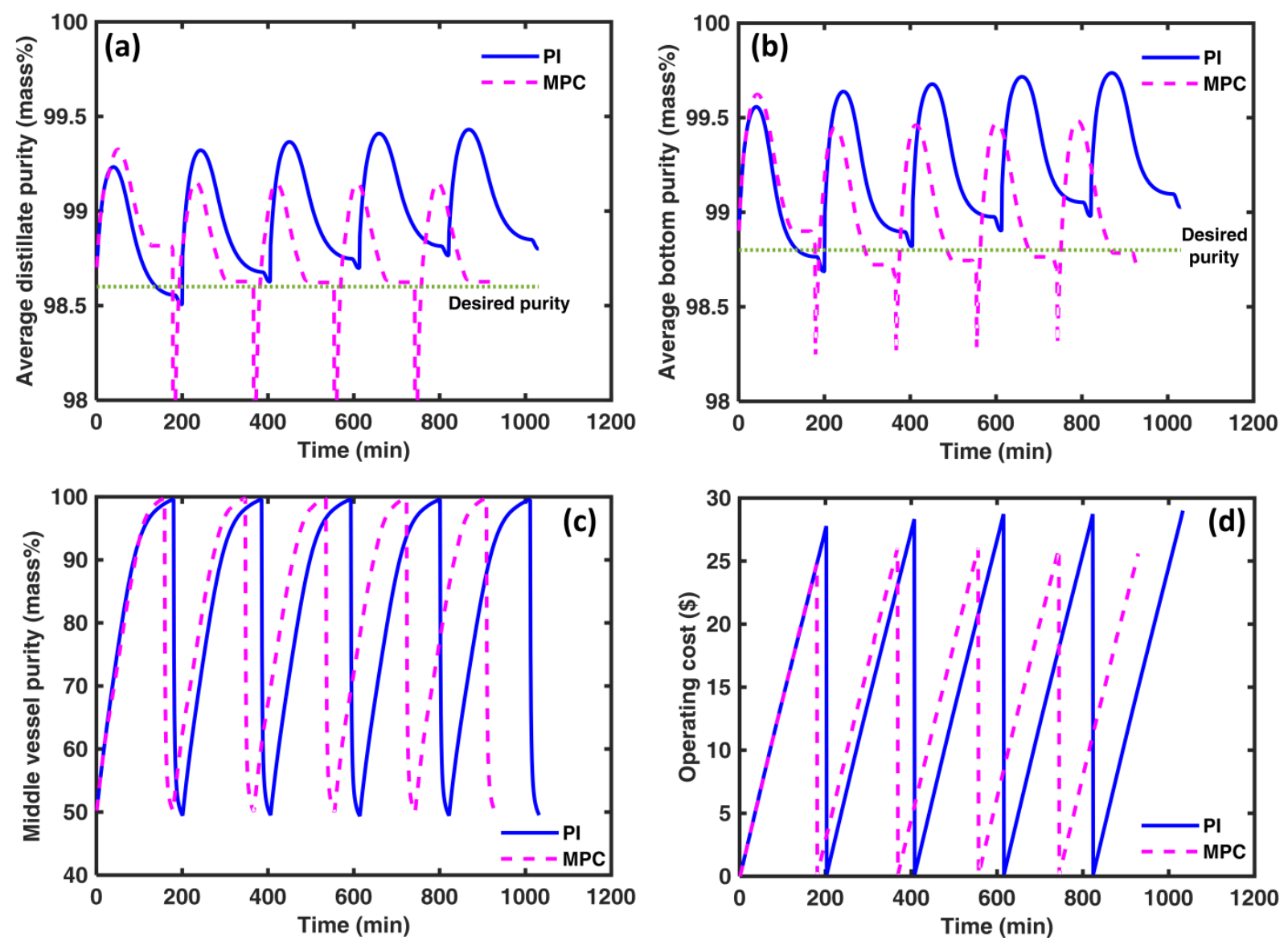

Figure 7- Variation of quality variables during five cycles.

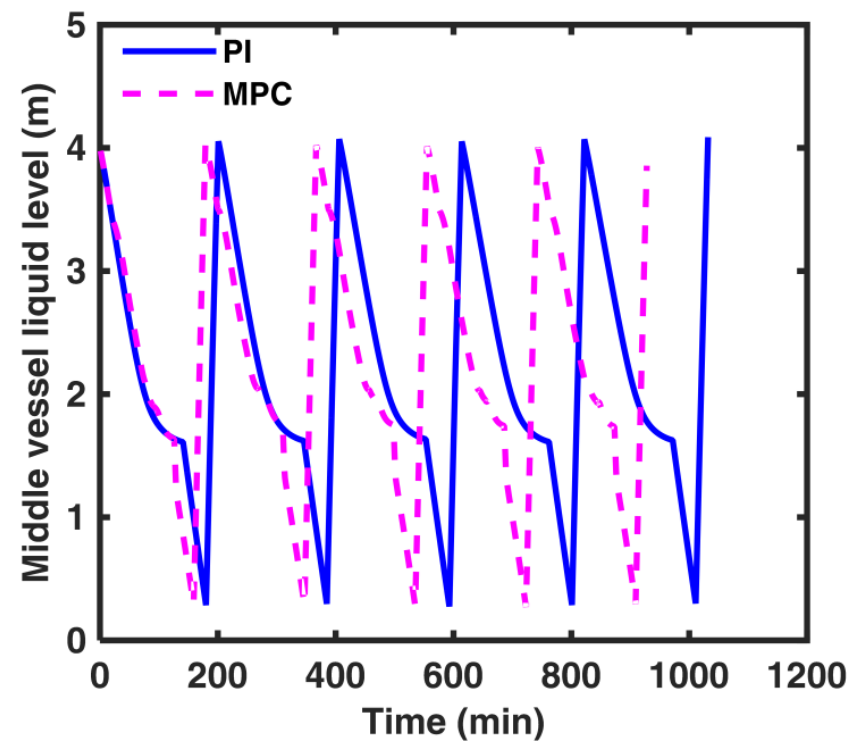

Figure 8- Liquid level in the middle vessel during semicontinuous cycles. 
Figure $7 \mathrm{c}$ shows the middle vessel purity and it illustrates that the MPC cycles are 10\% shorter than the multi-PI control configuration. The liquid level profile in the middle vessel during the cycles is shown in Figure 8. Finally, the operating cost of the process is shown in Figure 7d. The MPC has reduced the cost by $11.7 \%$ per cycle. This cost improvement is slightly due to avoiding the over purification of the products in each cycle and mainly due to a better operational policy that the MPC has computed for the semicontinuous process. Figure $7 \mathrm{~d}$ shows that the operating cost is essentially a linear function of time. Therefore, the MPC has reduced the cost by changing the operation of the process such that the separation can be achieved in a shorter cycle time.

The major improvement in the operation of the system achieved by the MPC is by changing the side stream flow rate pofile during the processing mode of the system. Two main policies for controlling the side stream flow rate have been utilized in previous studies for semicontinuous processes. The first policy is to maintain a constant side stream flow rate by having a flow controller on the stream with a fixed setpoint. This was found to be the most economic for the system and therefore used in this study. The second policy is the ideal side draw control strategy (Adams and Seider, 2008). In the ideal side draw control the setpoint of the side stream flow rate controller is adjusted by a cascade control which continually updates the setpoint to be equal to the amount of intermediate component entering the column via the middle vessel. With this control policy the side stream flow rate usually increases during the processing mode and hits a plateau by the end of that mode.

However, the MPC has found a more efficient policy for the side stream flow rate which shortens the process' cycle time. To explain the new policy, Figure 9 zooms in to the first full cycle of the semicontinuous process. The nominal PI and the MPC trajectories are shown to illustrate how the MPC has changed the operation of the system. The cycle starts from the processing mode and the vertical lines show the beginning of the charging and the discharging modes of the process for the MPC trajectories. Since the MPC cycles are 10\% shorter than the PI control configuration, the charging and discharging times are not at the same times for these two control configurations. 

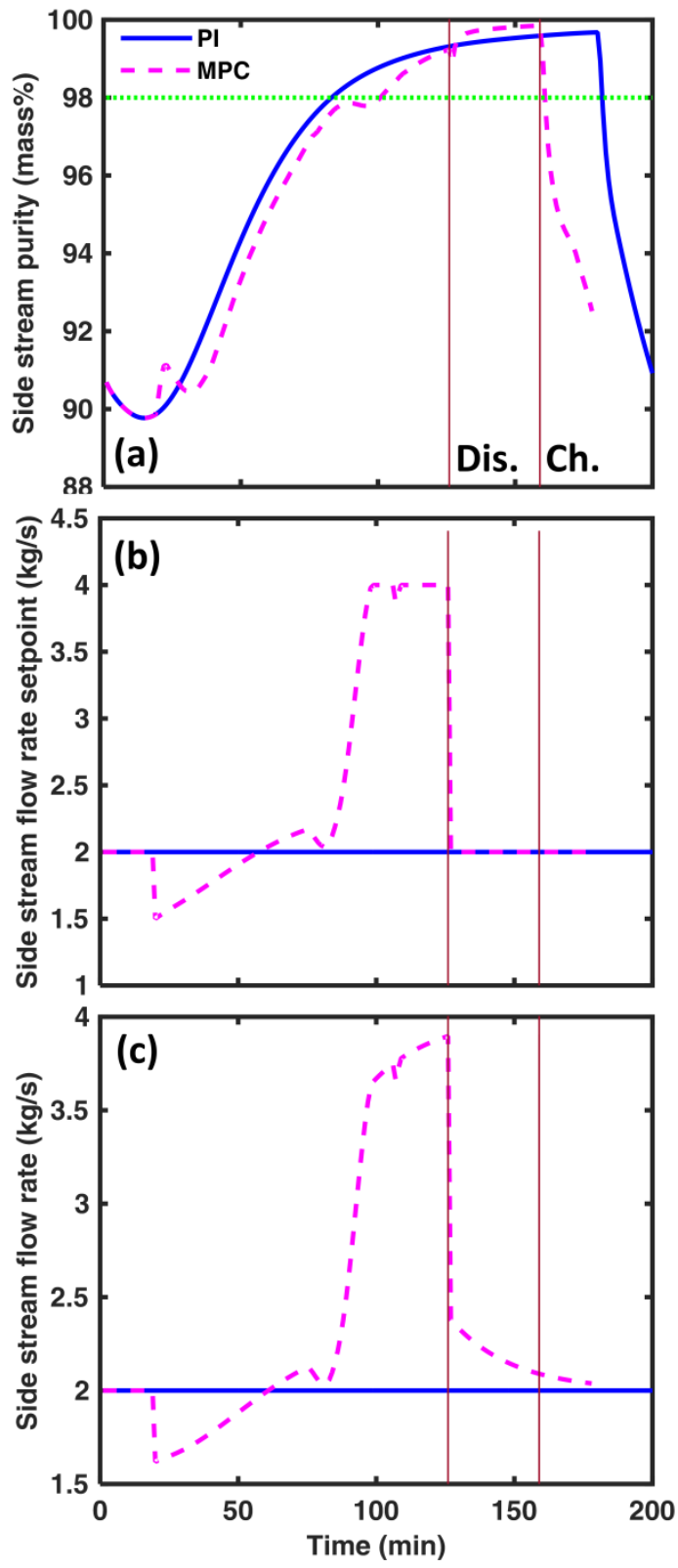

Figure 9- (a) Side stream purity, (b) controller setpoint, and (c) flow rate. The vertical lines corespond to the beginning of the discharging (Dis.) and charging (Ch.) modes of the process in the MPC configuration only.

As shown in Figure 9a, at the beginning of the processing mode, the purity of the intermediate component (toluene) in the side stream is lower than its specification (98 mass\%). During this period the MPC reduces the setpoint of the flow rate controller (Figure 9b) to withdraw less liquid from the column (Figure 9c). As this purity reaches the desired specification (around minute 90), the setpoint of the side stream flow rate controller is increased sharply and is put on 
its upper bound (Figure 9b). Consequently, the side stream flow rate is increased rapidly to its maximum flow rate (Figure 9c).

Recycling the side stream which is pure to the desired purity back to the middle vessle at the highest rate and feeding the tanks hold up at a higher rate back to the column has resulted in shortening the cycle time. Figure 9a shows that after the side stream purity has reached the desired specification, the profile has a higher slope with the MPC control than with the PI control.

At the beginning of the discharging mode the setpoint is brought back to the nominal value (Figure 9b). Figure 9c shows that the PI flow rate controller is fast enough to bring back the side flow rate to the nominal value without any ocillation by the end of the cycle. This is why faster PI controllers are more desirable in the MPC implementation. Another advantage of the MPC control policy is that by the end of the cycle, the purity of the side stream is higher than its coressponding value in the PI control system which results in a shorter subsequent cycle time.

It is interesting to mention that the side stream flow rate profile that the MPC has computed is simillar to the semicontinuous without middle vessel (SwoMV) configuration suggested by Meidanshahi and Adams (2015). The authors proposed that the semicontinuous process can achieve the same separation without the necessity of the middle vessel with a lower operational and direct costs. In the SwoMV configuration, there is no side stream withdrawal until the purity of the stream reaches above the desired specification. The MPC results show similar trend for the side stream flow rate.

Figure 10 shows the setpoint, the measurement and the manipulated variable of the reflux drum liquid level controller. In PI control configuration, the setpoint is set at $50 \%$ to keep the reflux drum level half full during the cycle. As the distillate flow rate decreases during the cycle (Figure 11c), the liquid level goes up in the reflux drum (Figure 10b). Therefore, the PI controller reduces the feed flow rate to the column (10c). Consequently the column is not working at its maximum capacity by the end of the cycle. However, MPC has fixed this drawback. In the MPC configuration, the reflux drum level setpoint is decreased and is set it to the lower bound during the processing mode (Figure 10a).

Initially, the reflux level jumps up since the distillate flow rate is increasing in that period (Figure 11c) which results in a dip in the feed flow rate to the column at about minute 20 (Figure 10c). However, as the distillate flow rate decreases during the cycle, the level goes down to the specified setpoint and therefore by the end of cycle the MPC can increase the feed flow rate to the column (Figure 10c). This increase in the feed flow rate occurs at about the same time as the MPC has increased the side stream flow rate to the column. These two synchronized effects result in shorter cycle time and lower operating cost. 

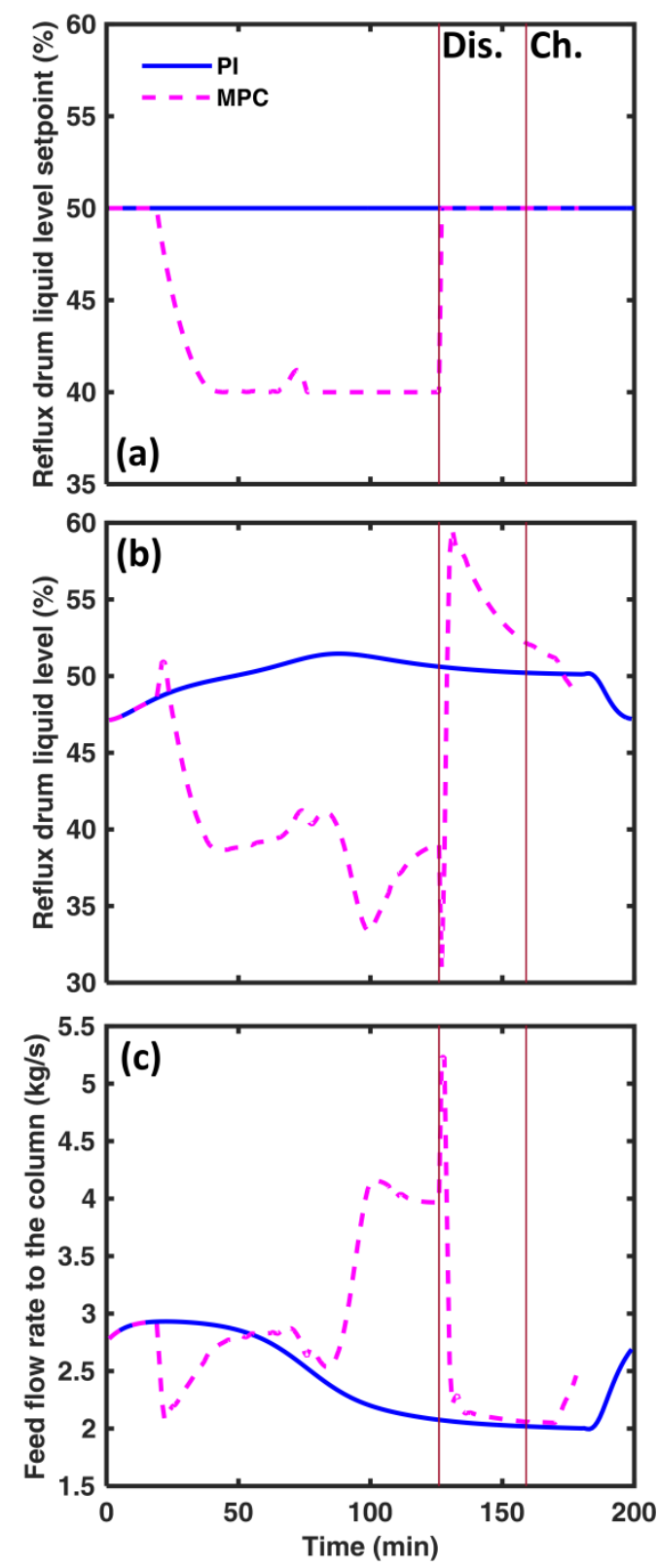

Figure 10- (a) Reflux drum's level controller setpoint, (b) level measurement, and (c) the manipulated variable (feed flow rate to the column).

Due to the decrease in the feed flow rate to the column at the beginning of the MPC cycle, the distillate stream purity has increased slightly (Figure 11b) and therefore the distillate flow rate is increased slightly during the processing mode (figure 11c). However, changes in the setpoint of distillate composition controller have resulted in distillate flow rate oscillation which is due to the tuning parameters of the PI controller. Especially, just before the beginning of the discharge mode, there is a short pick in distillate flow rate which is just at the same time that the feed flow rate to the column is at its maximum flow rate. This resulted in significant drop in the purity of 
the distillate stream during the discharging mode in the MPC configuration (Figure 11b), noting though that the distillate flow rate during this time is nearly zero and so the net effect on cumulative product quality is small. The MPC has tried to slightly increase the distillate composition controller setpoint to compensate for the purity drop in the discharging mode. The same behaviour is also observed in the bottoms composition controller as shown in Figure 12.
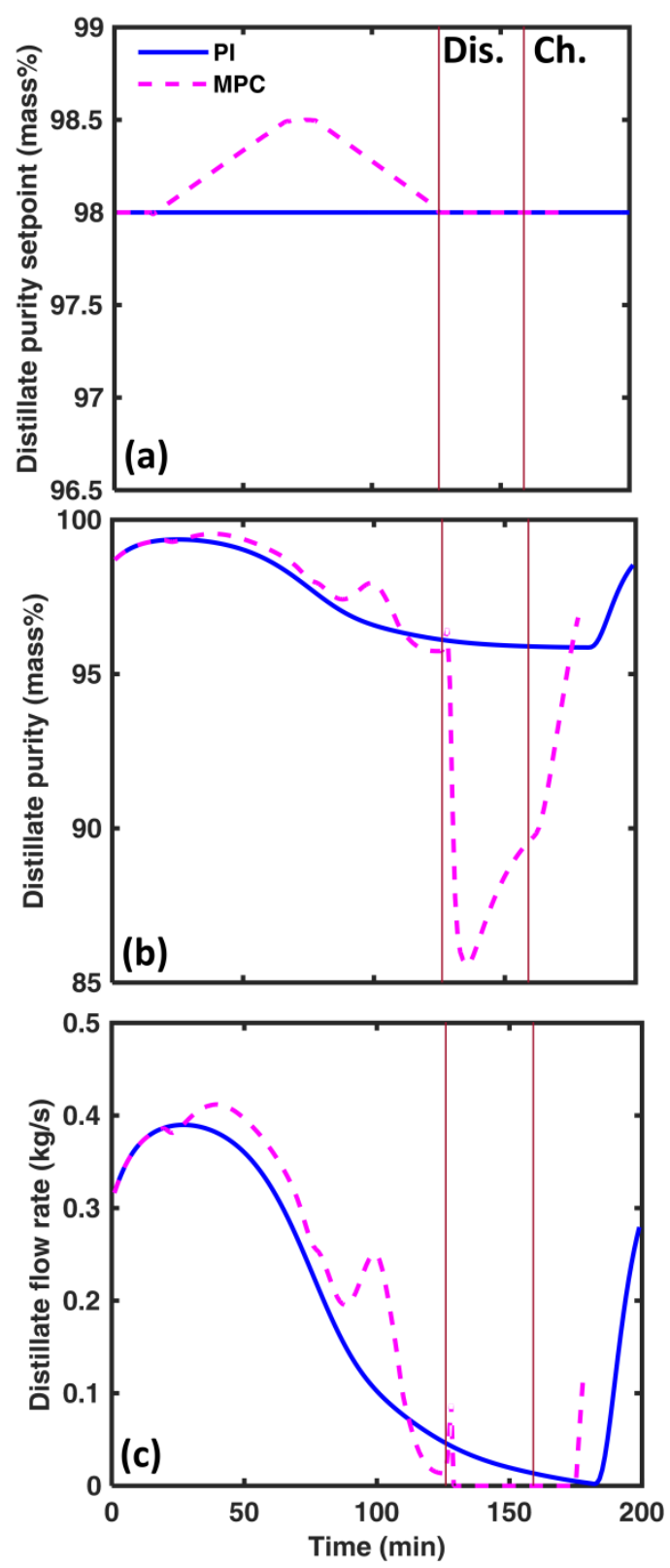

Figure 11- (a) Distillate composition controller's setpoint, (b) measurement and (c) manipulated variable. 

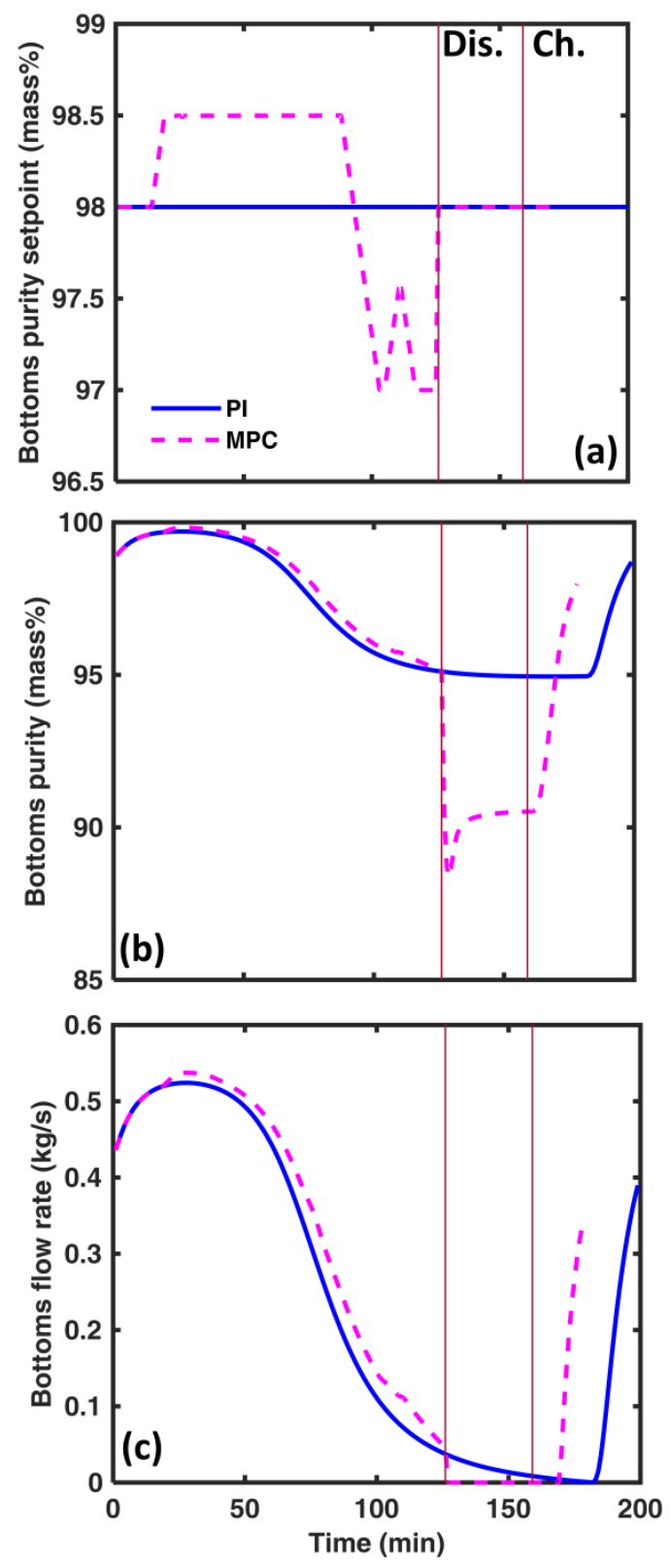

Figure 12- (a) Bottoms composition controller's setpoint, (b) measurement and (c) manipulated variable.

The changes in the sump's liquid level are shown in Figure 13. The setpoint is decreased to the lower bound. It is interesting to notice that this PI controller has a rather quick response to setpoint changes and the process variable follows the setpoint tightly. However, this fast response has resulted in a sharp increase in the reboiler heat duty at the beginning of the discharging mode as the liquid level is brought back very fast to its nominal value. It is important to note that the distillation model assumes that the reboiler system hardware is actually capable of delivering a rapid but brief spike of this magnitude. If this were not obtainable in reality, the 
system would take longer for the sump liquid level to return to $50 \%$ full but the general trends would be the same.
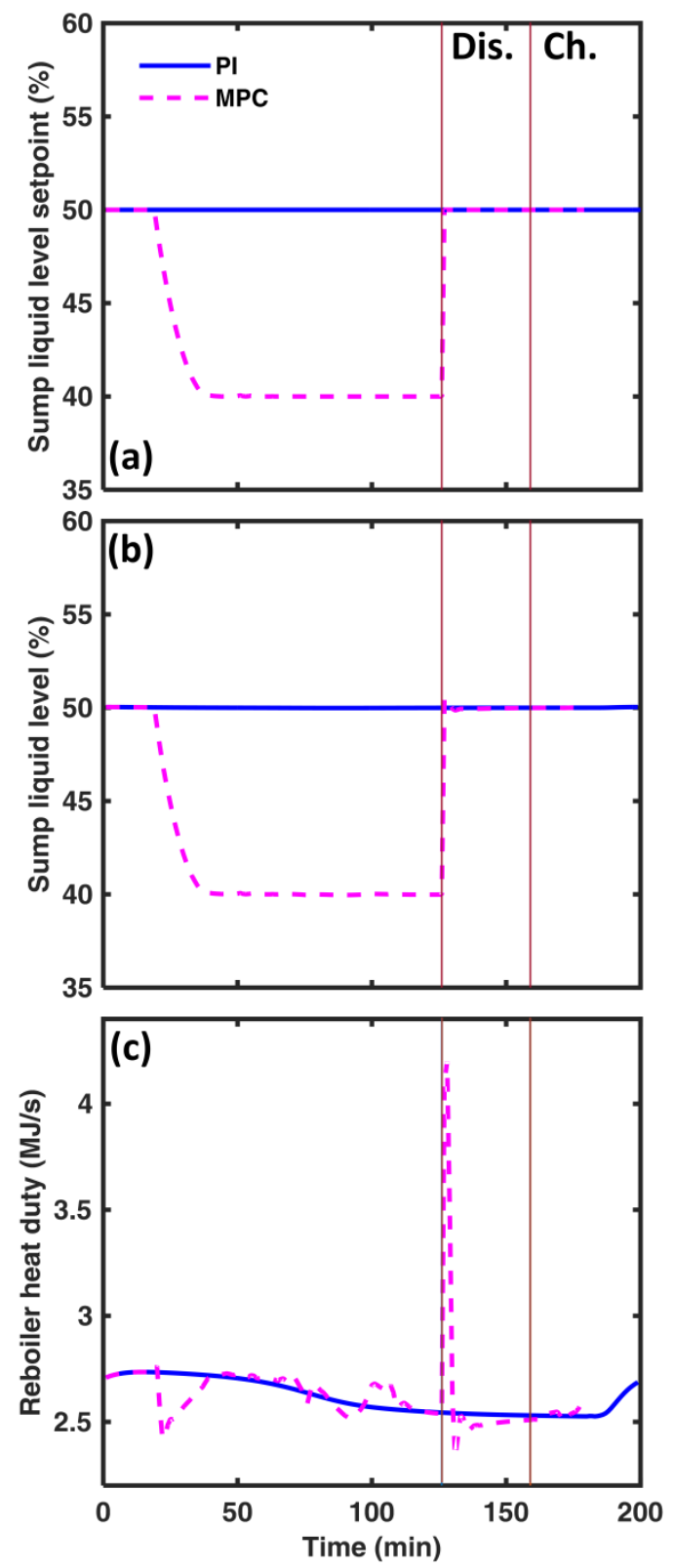

Figure 13- (a) Sump liquid level controller's setpoint, (b) measurement and (c) manipulated variable.

The manipulated variables of the PI controllers which are the distillate, bottoms, feed and side stream flow rates are plotted in Figure 14 during the five cycles of the semicontinuous system under the two control strategy studied in this work. Figure 14a and 14b show that the MPC has 
stabilized the cycles faster. Where in the PI control configuration the distillate and bottoms flow rates are gradually shrinking, these profiles look more stable with the MPC control.
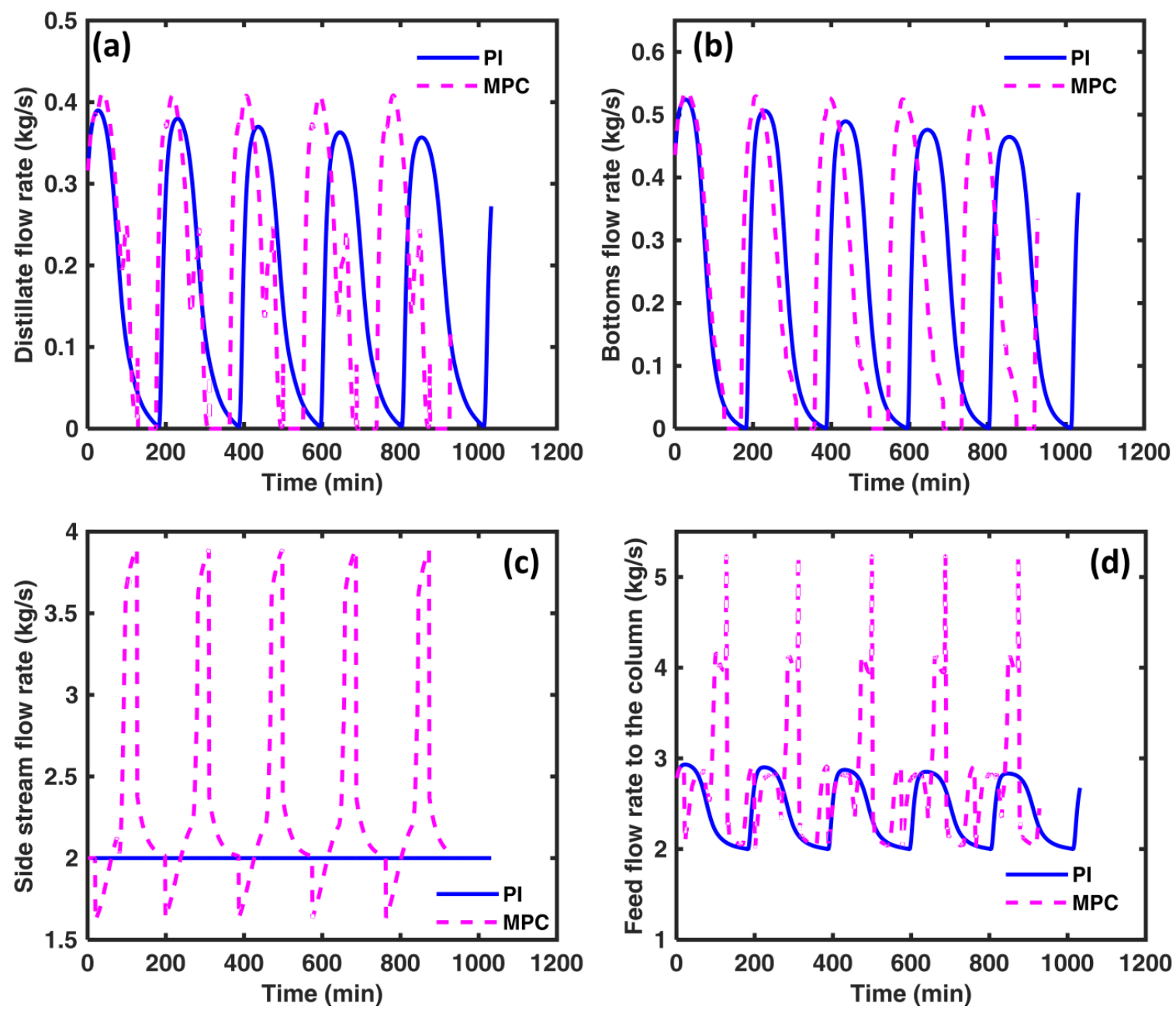

Figure 14- Manipulated variables of the PI controllers during the five cycles of multi-PI and cascaded MPC with PI control configurations.

Finally, in order to ensure safe and stable operation, operational constraints are considered in the MPC. These constraints are shown in Figure 15. Figure 15a and 15b show the liquid levels in the reflux drum and sump, respectively. These Figures show that the levels are kept in the desired ranges (10-90\% of the vessel heights) during the cycles. Figure $15 \mathrm{c}$ and $15 \mathrm{~d}$ show the vapour velocities at top (tray number 5) and bottom (tray number 20) of the column, respectively. The vapour velocities are kept in between the limits to avoid weeping and flooding of the column throughout the cycles. The spikes at the end of the processing modes in the vapour velocity profiles are due to the sharp increase in the reboiler heat duties and consequently increased 
vapour flow rate in the column. However, the vapour velocities are still slightly below the maximum allowable velocity.
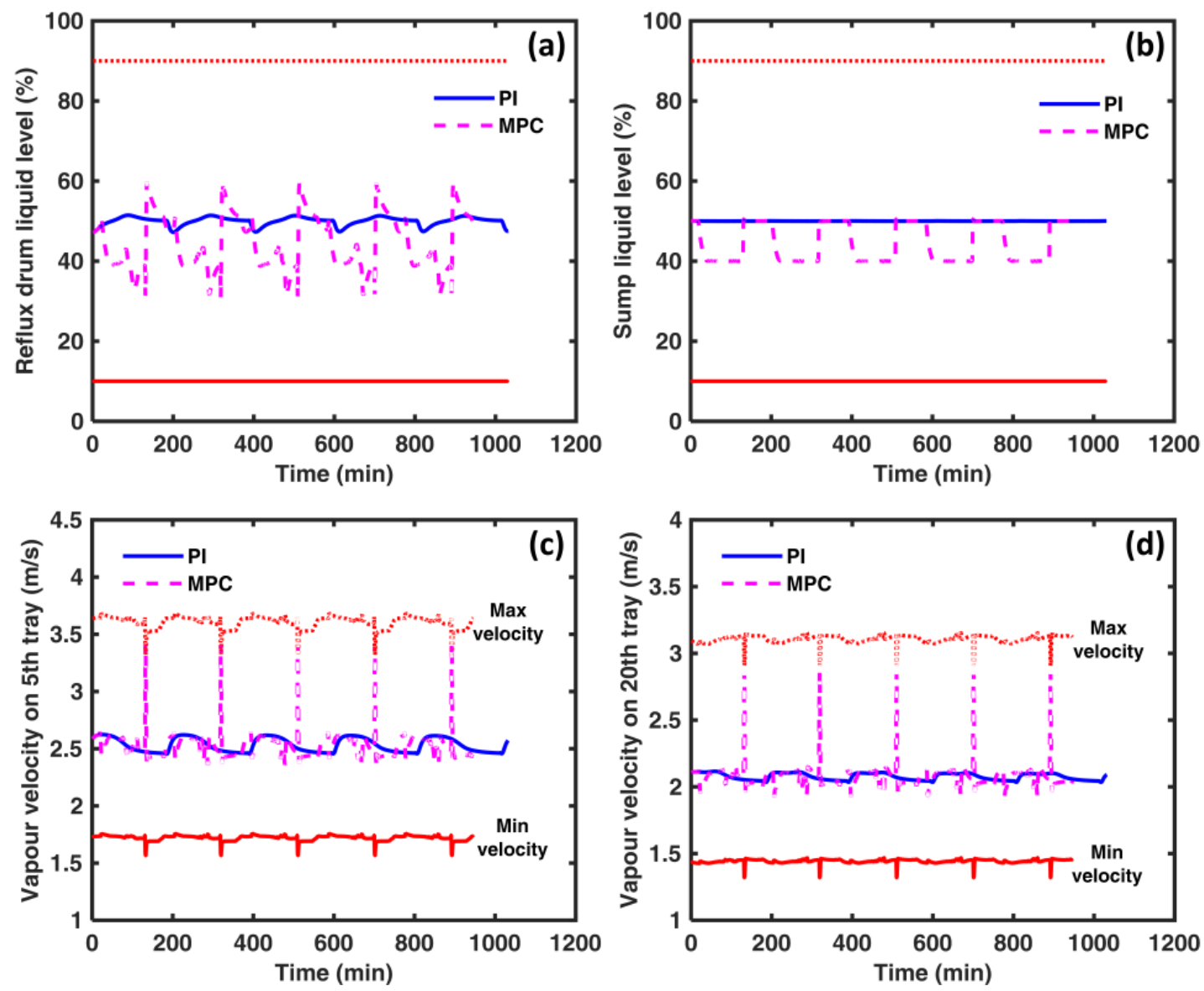

Figure 15- Operational constraints on the process.

For the last remark, it should be noted that MPC calculation time at each sampling instant is fairly shorter than the sampling time of the process. This ensures the applicability of the method to the process. One advantageous of the SQMPC method is its fast computational time at each sampling instance. The optimization problem is formulated as a convex, quadratic programming problem which can be solved fast while guaranteeing optimality of the solutions. The SQMPC computational time at each sampling instance took on average 0.6549 seconds in this study (with minimum calculation time of 0.0296 seconds and maximum 11.34 seconds, on a $3.4 \mathrm{GHz}$, Intel, Core i7, 64-bit machine). This computational time is smaller than the sampling time of the process which was 60 seconds and ensures applicability of the method on the process. 


\subsection{Case 2: Change in the initial feed composition}

In this case the performance of the MPC with change in the initial feed composition is studied. The linear model and all parameters are kept constant except the $\boldsymbol{M}$ matrix. In this case heavier penalties are considered for the average distillate and bottoms purities as shown in Table 4. In this case, from the second cycle of the semicontinuous process, the mass fraction of the intermediate component is increased by $10 \%$. The responses of the PI and the cascaded MPC control configurations are examined.

Table 4- Tuning parameters of the MPC for case 2.

\begin{tabular}{cccc}
\hline$m_{1}$ & $m_{2}$ & $m_{3}$ & $m_{4}$ \\
\hline 50 & 5000 & 5000 & 1000 \\
\hline
\end{tabular}
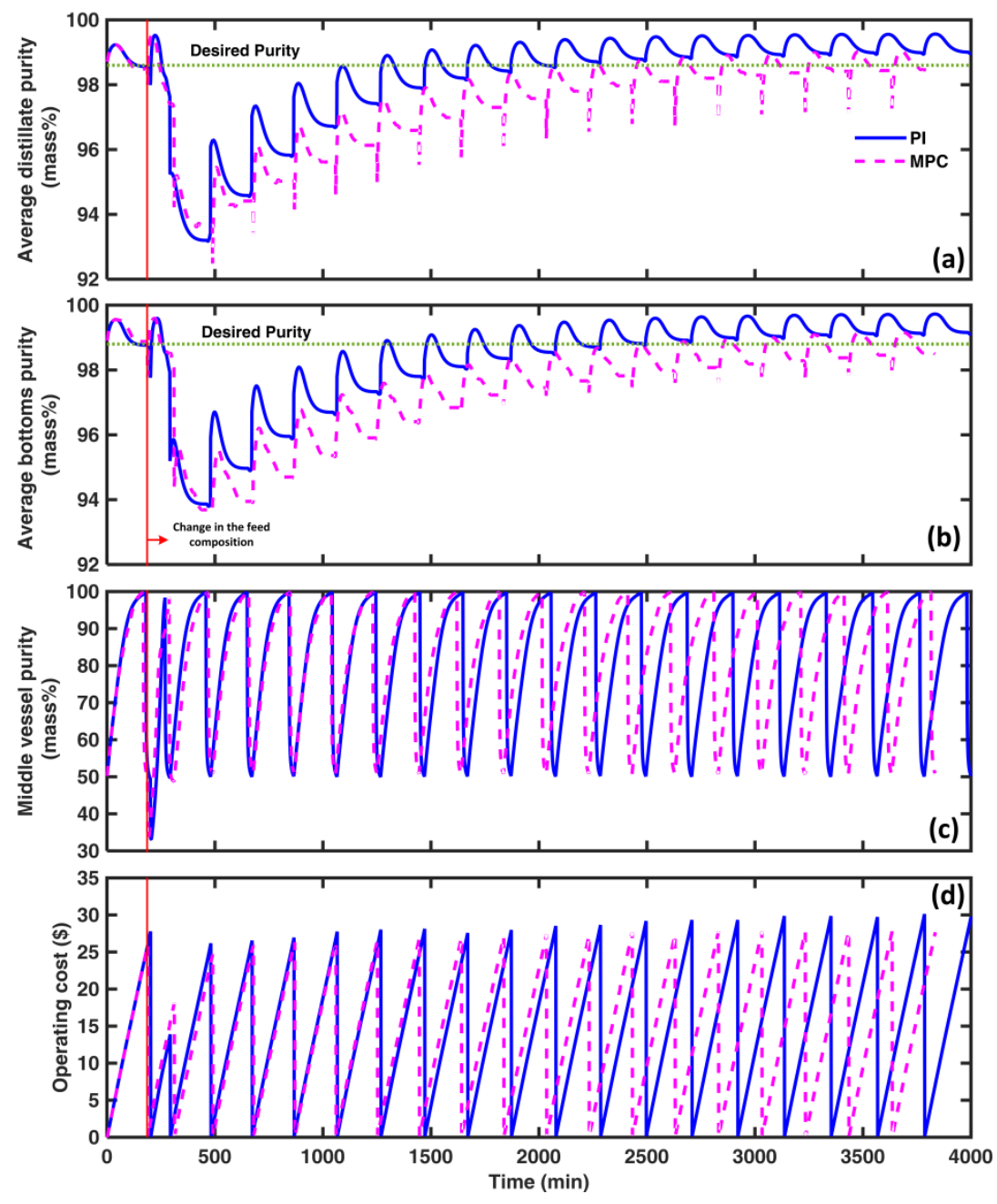

Figure 16- Quality variables profiles for twenty cycles with the change in the feed composition. 
The quality variable profiles are shown in Figure 16. The behaviour of the MPC in handling the change is similar to the PI control. However, the MPC has brought all the product purities close to the desired specifications while having a shorter cycle times and consequently $8.17 \%$ lower operational cost per cycle compared to the PI configuration. The corresponding inputs to the system are shown in Figure 17. The MPC input profiles follow the same trend as the previous case study. The importance of this case study is to show that the MPC can handle step changes in the feed stock conditions of the process even without an updated model.

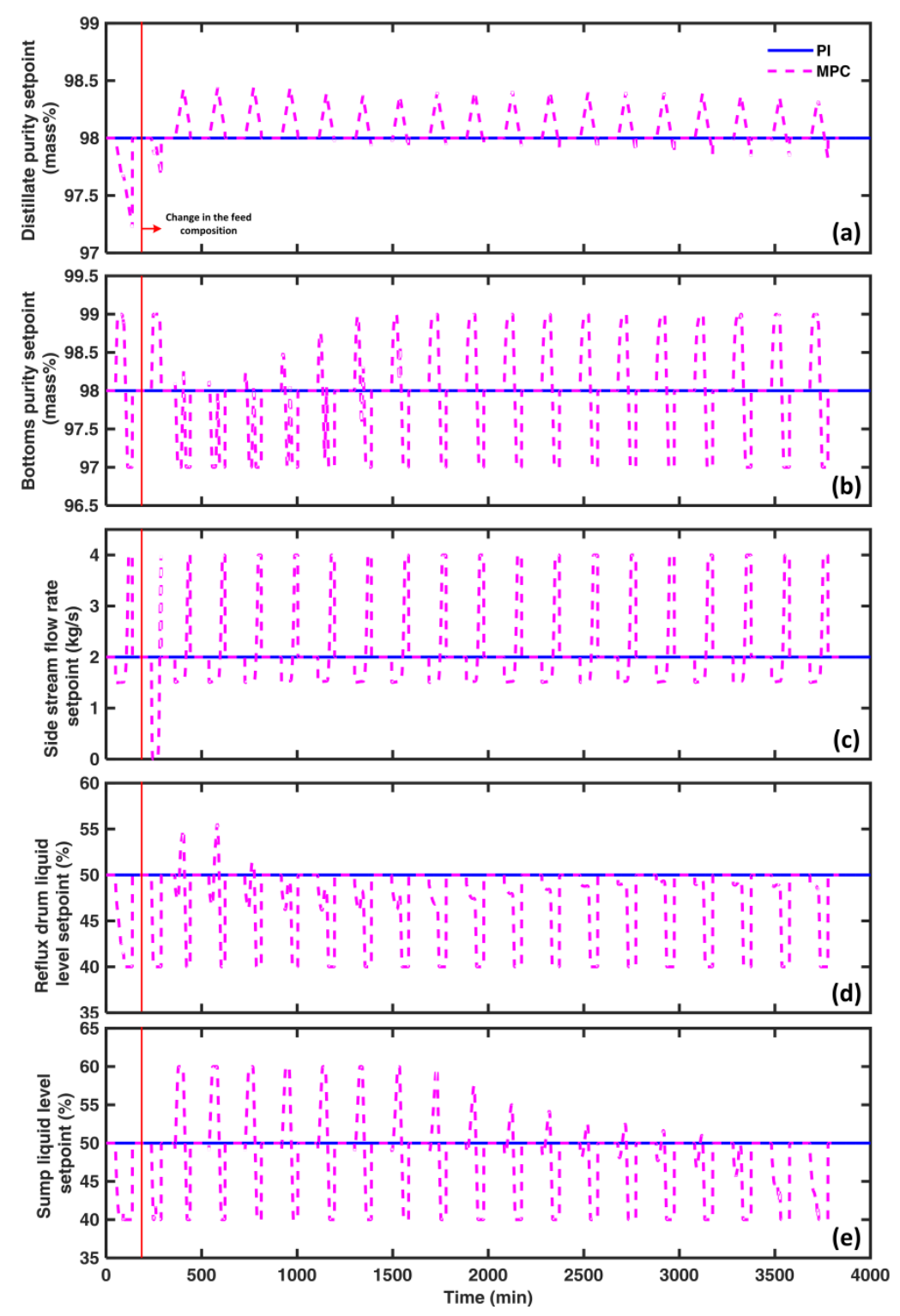

Figure 17- PI setpoints for the studied control configurations with $10 \%$ change in the feed stock composition implemented to the process from the second cycle. 


\section{Conclusion}

In this study implementation of the MPC on a semicontinuous process is studied for the first time. The objective of the MPC is to reduce the operational costs of the process while maintaining all product purities. Direct and cascaded MPC configurations are studied and it is concluded that the cascaded MPC with PI control loops is a better configuration for the semicontinuous process. Subsequently, the subspace identification method is adopted to identify a linear, state-space model of the process. It is shown that the model can predict the dynamics of the process sufficiently well for control purposes. Finally, a shrinking horizon SQMPC method is devised to compute the inputs to the process which are the setpoints of the PI controllers. The MPC results show up to $11.7 \%$ reduction in the operational cost of the process per cycle. This improvement is the result of $10 \%$ reduction in the cycle time of the semicontinuous process with the MPC strategy. Higher cost improvements could potentially be obtained with optimum tuning parameters of the MPC.

In multi-PI control configuration, the distillate and bottoms flow rates decrease during the cycle as the concentrations of light and heavy boiling point components decrease in the middle vessel. Subsequently, the feed flow rate to the column is decreased. Meanwhile, the side stream flow rate is either kept constant or is increased or decreased (depending on the design of the system) during the cycle until the flow rate hits the plateau by the end of the cycle. However, the MPC has changed this operational policy and found a shorter path to purify the mixture.

In the cascaded MPC with multi-PI configuration, the feed and the side stream flow rates are decreased at the beginning of the processing mode. This will reduce recycling at the beginning of the processing mode and will lessen the mixing of the purified side stream with the un-purified middle vessel holdup. Subsequently, as the purity of the side stream reaches the desired specification, the feed flow rate to the column and the side stream flow rate are increased to accelerate the separation. This operational policy computed with the MPC agrees well with the SwoMV configuration which was already shown more economical than the semicontinuous system for a range of operations.

Furthermore, it was observed that the MPC can dampen the effect of the PI tuning parameters and stabilize the process faster. However, the effect of the PI tuning parameters on the performance of the MPC is the focus of future work. Also, it is suggested that the tuning parameters of the PI controllers are included as inputs in the MPC algorithm so they can be adjusted as the cycle proceeds.

Finally, the performance of the MPC is examined for a case with changes in the feed stock composition. It is observed that MPC can maintain its superior performance compared to the PI control configuration in this case as well.

The inclusion of the charging and discharging modes of operation in the MPC is also the subject of ongoing research. This will probably result in better control and economics for the system because the MPC will not have to reset at the start of each cycle, forcing the inputs to return to 
their nominal values each cycle and requiring the observer to re-converge each cycle. Instead, the information from previous cycles will be carried forward to better inform future cycles.

\section{Acknowledgements}

The authors would like to graciously acknowledge the Ontario Trillium Scholarships program for their financial support of this project.

\section{Nomenclature}

$\begin{array}{ll}F & \text { Flow rate, }(\mathrm{kg} / \mathrm{s}) \\ \boldsymbol{M} & \text { Penalty matrix on quality variables } \\ \boldsymbol{P} & \text { Penalty matrix on input moves } \\ \boldsymbol{q} & \text { Quality variable } \\ \mathrm{Q}_{\text {condenser }} & \text { Condenser heat duty, }(\mathrm{kJ} / \mathrm{s}) \\ \mathrm{Q}_{\text {reboiler }} & \text { Reboiler heat duty, }(\mathrm{kJ} / \mathrm{s}) \\ t_{f} & \text { Final time, }(\mathrm{s}) \\ \boldsymbol{u} & \text { Input variables } \\ \boldsymbol{v} & \text { Candidate input trajectory } \\ V & \text { Vapour velocity, }(\mathrm{m} / \mathrm{s}) \\ \mathrm{v}_{1} & \text { Cost of condenser heat duty, }(\$ / \mathrm{kJ}) \\ \mathrm{v}_{2} & \text { Cost of reboiler heat duty, }(\$ / \mathrm{kJ}) \\ \boldsymbol{x} & \text { State variables } \\ \tilde{\boldsymbol{x}} & \text { Subspace states } \\ x_{B}^{\text {dis }} & \text { Purity of benzene in the distillate stream, (mass \%) } \\ x_{X}^{b o t} & \text { Purity of } o \text {-xylene in the bottom stream, (mass \%) } \\ \boldsymbol{y} & \text { Output variables } \\ & \end{array}$

Abbreviations

DAE

Differential algebraic equation

MPC

Model predictive control 


$\begin{array}{ll}\text { MVCC } & \text { Middle vessel continuous distillation column } \\ \text { PI } & \text { Proportional integral } \\ \text { PID } & \text { Proportional integral derivative } \\ \text { PML } & \text { Process model library } \\ \text { PRBS } & \text { Pseudo-random binary sequence } \\ \text { SQMPC } & \text { Subspace quality model predictive control }\end{array}$

\section{References}

Adams II TA, Seider WD. Semicontinuous distillation for Ethyl Lactate production. AIChE J.2008; 54(10): 2539-2552.

Adams II TA, Pascall A. Semicontinuous thermal separation systems. Chem. Eng. Technol. 2012; 35(7): 1153-1170.

Aumi S, Mhaskar P. Integrating data-based modeling and nonlinear control tools for batch process control. AIChE Journal 2012; 58(7): 2105-2119.

Barolo M, Papini CA. Improving dual composition control in continuous distillation by a novel column design. AIChE Journal 2000; 46(1): 146-159.

Bezzo F, Micheletti F, Muradore R, Barolo M. Using MPC to control middle-vessel continuous distillation columns. Journal of Process Control 2005; 15: 925-930.

Corbett B, Mhaskar P. Subspace identification for data-driven modeling and quality control of batch processes. AIChE Journal 2016; 62(5): 1581-1601.

Dunnebier G, Pantelides CC. Optimal design of thermally coupled distillation columns. Ind. Eng. Chem. Res. 1999; 38: 162-176.

Huang H, Riggs JB. Including levels in MPC to improve distillation control. Ind. Eng. Chem. Res. 2001; 41: 4048-4053.

Jana AK. Heat integrated distillation operation. Applied Energy 2010; 87: 1477-1494.

Meidanshahi V, Adams II TA. A new process for ternary separations: semicontinuous distillation without a middle vessel. Chem. Eng. Res. Des. 2015; 93: 100-112.

Meidanshahi V, Adams II TA. Integrated design and control of semicontinuous distillation systems utilizing mixed integer dynamic optimization. Computers and Chemical Engineering 2016; 89: 172-183.

Mersmann A, Kind M, Stichlmair J. Thermal separation technology, principles, methods, process design. Springer Heidelberg Dordrecht, London New York, 2011.

Moonen M, De Moor B. On- and off-line identification of linear state-space models. Int. J. Control. 1989; 49(1): 219-232. 
Pascall A, Adams II TA. Semicontinuous separation of Dimethyl Ether (DME) produced from biomass. The Canadian Journal of Chemical Engineering 2013; 9999: 1-21.

Phimister JR, Seider WD. Semicontinuous, middle-vessel distillation of ternary mixtures. AIChE J. 2000; 46 (8): 1508-1520.

Qin SJ. An overview of subspace identification. Computers and Chemical Engineering 2006; 30: 1502-1513.

Rewagad RR, Kiss AA. Dynamic optimization of a dividing-wall column using model predictive control. Chemical Engineering Science 2012; 68: 132-142.

Sultana A, Kumar A. Optimal siting and size of bioenergy facilities using geographic information system. Applied Energy 2012; 94: 192-201.

Towler G, Sinnott R. Chemical engineering design: Principles, practice and economics of plant and process design. 2nd ed. Massachusetts: Butterworth-Heinemann, 2012.

Wijesekera KN, Adams II TA. Semicontinuous distillation of quaternary mixtures using one distillation column and two integrated middle vessels. Ind. Eng. Chem. Res. 2015a; 54: 5294-5306.

Wijesekera KN, Adams II TA. Semicontinuous distillation of quintenary and N-ary mixtures. Ind. Eng. Chem. Res. 2015b; 54: 12877-12890.

Yildirim O, Kiss AA, Kenig EY. Dividing wall columns in chemical process industry: A review on current activities. Separation and Purification Technology 2011; 80: 403-417. 